(c) 2006 International Press

Adv. Theor. Math. Phys. 10 (2006) 603-656

\title{
BPS microstates and the open topological string wave function
}

\author{
Mina Aganagic ${ }^{1}$, Andrew Neitzke ${ }^{2}$, and Cumrun Vafa ${ }^{2}$ \\ ${ }^{1}$ University of California, Berkeley, CA 94720, USA \\ mina@socrates.berkeley.edu \\ ${ }^{2}$ Jefferson Physical Laboratory, Harvard University, Cambridge, \\ MA 02138, USA \\ neitzke@post.harvard.edu; vafa@string.harvard.edu
}

\begin{abstract}
It has recently been conjectured that the closed topological string wave function computes a grand canonical partition function of Bogomol'nyiPrasad-Sommerfield (BPS) black hole states in four dimensions: $Z_{\mathrm{BH}}=$ $\left|\psi_{\text {top }}\right|^{2}$. We conjecture that the open topological string wave function also computes a grand canonical partition function, which sums over black holes bound to BPS excitations on D-branes wrapping cycles of the internal Calabi-Yau: $Z_{\mathrm{BPS}}^{\text {open }}=\left|\psi_{\text {top }}^{\text {open }}\right|^{2}$. This conjecture is verified in the case of Type IIA on a local Calabi-Yau three-fold involving a Riemann surface, where the degeneracies of BPS states can be computed in $q$-deformed two-dimensional Yang-Mills theory.
\end{abstract}

e-print archive: http://lanl.arXiv.org/abs/hep-th/0504054 


\section{Contents}

1. Introduction 605

$\begin{array}{lll}\text { 2. The closed string case } & 607\end{array}$

2.1. A solvable example $\quad 608$

$\begin{array}{ll}\text { 3. Revisiting the closed string theory } & 610\end{array}$

$\begin{array}{ll}\text { 3.1. Open vs. closed } & 612\end{array}$

3.2. The attractor mechanism and ghost D-branes 613

3.3. Why $2 g-2$ asymptotic infinities 614

4. The quantum mechanics of open strings 615

4.1. Canonical quantization in Chern-Simons 615

4.2. Background dependence for the open topological string $\quad 618$

$\begin{array}{ll}\text { 5. The open string conjecture } & 619\end{array}$

5.1. Calabi-Yau spaces with branes and BPS particles $\quad 620$

$\begin{array}{ll}\text { 5.2. The conjecture } & 621\end{array}$

5.3. Adding magnetic charges $\quad 622$

5.4. Multiple Lagrangian branes $\quad 624$

$\begin{array}{ll}\text { 6. A solvable example } & 625\end{array}$

$\begin{array}{ll}\text { 6.1. } & \text { Specializing to genus zero }\end{array}$

6.2. Large $N$ factorization on $\mathcal{O}(-p) \oplus \mathcal{O}(p-2) \rightarrow$ $\mathbb{C P}^{1} \quad 628$

6.3. Summing open D2-branes on $\mathcal{O}(-1) \oplus \mathcal{O}(-1) \rightarrow$ $\mathbb{C P}^{1}$

$\begin{array}{ll}\text { Acknowledgments } & 636\end{array}$ 
$\begin{array}{lll}\text { Appendix A. Group theory } & 636\end{array}$

Appendix B. The $q$-deformed 2-d Yang-Mills theory 639

$\begin{array}{lll}\text { Appendix C. The disc wave function } & 642\end{array}$

Appendix D. Factorization $\quad 644$

D.1. Coupled representations $\quad 644$

$\begin{array}{lll}\text { D.2. Branching rules } & 647\end{array}$

$\begin{array}{lll}\text { D.3. } & \text { Factorization of } S_{\mathcal{P} \mathcal{Q}} & 648\end{array}$

D.4. Factorization of
amplitudes

$\begin{array}{ll}\text { References } & 654\end{array}$

\section{Introduction}

The connection between topological strings and four-dimensional BPS black holes has been studied in recent years [1-3], leading to a conjecture [4] that identifies the mixed grand canonical partition function of BPS black hole states with the squared norm of the topological string wave function: $Z_{\mathrm{BH}}=\left|\psi_{\text {top }}\right|^{2}$. This conjecture has been checked for certain Calabi-Yau three-folds [5-7]; see also the recent related work [8-10]. It is natural to ask how the conjecture generalizes to the case of open topological strings. Our primary aim in this paper is to advance a conjecture about what the open topological string counts, and to check it in the case of certain non-compact Calabi-Yau spaces.

We will mainly concentrate on the Type IIA superstring (and correspondingly the topological A model) on a non-compact Calabi-Yau three-fold. In the closed string context, one defines the mixed black hole ensemble by fixing the number of D4 and D6-branes (magnetic charges) whereas summing over all possible numbers of D2 and D0-branes bound to them (electric charges), weighed by chemical potentials; this was the setup investigated in refs. $[5,6]$. In our case, the Type IIA background will additionally include a finite number of "background" D4-branes, which wrap Lagrangian 3-cycles of the Calabi-Yau and fill a 1+1 dimensional subspace of Minkowski spacetime. In the presence of these background D4-branes one gets a gauge theory in $1+1$ dimensions, containing new BPS states. The role of the electric 
charges is played by open D2-branes, wrapped on holomorphic discs ending on the Lagrangian 3-cycles, whereas the magnetic charges are domain walls in the $1+1$ dimensional theory. We conjecture that the full topological string amplitude, including contributions from open strings, is counting degeneracies of these BPS states, bound to D6, D4, D2 and D0-branes:

$$
Z_{\mathrm{BPS}}^{\text {open }}=\left|\psi_{\text {top }}^{\text {open }}\right|^{2} \text {. }
$$

Here, $Z_{\mathrm{BPS}}^{\text {open }}$ is the partition function of a mixed grand canonical ensemble; in this ensemble the D6 and D4-brane charges, as well as the domain wall charge, are fixed (and related to the real part of the topological string moduli), whereas chemical potentials are turned on for the D2 and D0-branes (giving the imaginary parts of the moduli), including the open D2-branes.

Our proposal is necessarily more tentative than the one given in ref. [4], because one of the major planks supporting the conjecture there is missing here: the large-charge macroscopic/gravitational description of the BPS states we are counting has not been studied, nor has the analog of the attractor mechanism for these states, so we do not even have a classical derivation of the entropy. Further investigations in this direction would be extremely useful to check our conjecture.

Although we do not understand the macroscopic description of these BPS states, we can still compare $\left|\psi_{\text {top }}^{\text {open }}\right|^{2}$ to a partition function computed from their microscopic description, in cases where such a description is available. In this article, we use such a description to check our proposal on a particular non-compact Calabi-Yau space supporting a compact Riemann surface. This case was previously discussed in refs. [5,6] where the closed string conjecture was verified. We find that our conjecture also holds in this case.

The organization of this article is as follows. In Section 2, we review the conjecture in the closed string case and review its confirmation in the context of local Riemann surfaces inside a Calabi-Yau. In Section 3, we explain the unexpected appearance of open topological string amplitudes in ref. [6], reinterpreting them in terms of purely closed topological strings along the lines of the original conjecture [4]. In Section 4, we discuss the wave function nature of the open topological string. In Section 5, we introduce additional branes in our physical string background and state our main conjecture. In Section 6, we check the conjecture in the context of a local Calabi-Yau geometry near a Riemann surface with Lagrangian D-branes included. Most of the computations are relegated to the appendices: In Appendix A, we fix some group theory conventions and review some basic group theory facts. In Appendix B, we review the $q$-deformed Yang-Mills theory in two dimensions and the computation of its amplitudes by gluing, including insertion of some eigenvalue freezing operators important for this article. In Appendix C, 
we express the wave function of $q$-deformed $2 d$ Yang-Mills on the disc in terms of theta functions. Finally, Appendix D discusses many issues related to the large $N$ limit of our computations, and the factorization of the BPS partition function at large $N$ in terms of topological and anti-topological contributions. In particular, we give a physical explanation of the factorization of the $q$-deformed Yang-Mills amplitudes in the large $N$ limit.

\section{The closed string case}

In ref. [4], a duality was conjectured which relates counting of microstates of supersymmetric black holes, which arise in compactification of Type II string theory on a Calabi-Yau three-fold $X$ and closed topological string theory on $X$. In this section, we review this conjecture and one case in which it has been explicitly checked.

Consider Type IIA on $X \times \mathbb{R}^{3,1}$. One can obtain charged BPS black holes in $\mathbb{R}^{3,1}$ by wrapping D6, D4, D2 and D0-branes over holomorphic cycles in $X$. The charges of the black hole are determined by the choice of holomorphic cycles; the intersection pairing in $X$ gives rise to the electric-magnetic pairing in $\mathbb{R}^{3,1}$, and we refer to D6 and D4-brane charges as "magnetic" whereas D2 and D0-brane charges are "electric." Then one can define a mixed ensemble of BPS black hole states by fixing the D6 and D4-brane charges $Q_{6}, Q_{4}$, and summing over D2 and D0-brane charges with fixed chemical potentials $\varphi_{2}, \varphi_{0}$. One can write a partition function for this ensemble,

$$
Z_{\mathrm{BH}}\left(Q_{6}, Q_{4}, \varphi_{2}, \varphi_{0}\right)=\sum_{Q_{2}, Q_{0}} \Omega_{Q_{6}, Q_{4}, Q_{2}, Q_{0}} \mathrm{e}^{-Q_{2} \varphi_{2}-Q_{0} \varphi_{0}} .
$$

Here $\Omega_{Q_{6}, Q_{4}, Q_{2}, Q_{0}}$ is the contribution from BPS bound states with fixed D-brane charge.

The conjecture of ref. [4] is that

$$
Z_{\mathrm{BH}}\left(Q_{6}, Q_{4}, \varphi_{2}, \varphi_{0}\right)=\left|\psi_{\text {top }}\left(g_{\text {top }}, t\right)\right|^{2},
$$

where $\psi_{\text {top }}\left(g_{\text {top }}, t\right)$ denotes the A model topological string partition function, evaluated at the topological string coupling ${ }^{1}$

$$
g_{\mathrm{top}}=\frac{4 \pi i}{i \frac{\varphi_{0}}{\pi}+Q_{6}}
$$

and Kähler parameter

$$
t=\frac{1}{2} g_{\mathrm{top}}\left(i \frac{\varphi_{2}}{\pi}+Q_{4}\right)
$$

\footnotetext{
${ }^{1} Q_{4}$ is naturally a class in $H_{4}(X, \mathbb{Z})$, which we are relating to $t \in H^{2}(X, \mathbb{C})$, and $Q_{6}$ is naturally a class in $H_{6}(X, \mathbb{Z})$, which we are relating to $H^{0}(X, \mathbb{C})=\mathbb{C}$.
} 
The real parts of the parameters (2.3) and (2.4) are dictated by the "attractor mechanism" of $\mathcal{N}=2, d=4$ supergravity [11,12], which relates the moduli of $X$ near a black hole horizon to the black hole charges.

One can (at least formally) invert the relation (2.2) to recover the microcanonical degeneracies $\Omega$ from $\left|\psi_{\text {top }}\right|^{2}$, via the integral formula

$$
\Omega_{Q_{6}, Q_{4}, Q_{2}, Q_{0}}=\int d \varphi_{2} d \varphi_{0} \mathrm{e}^{Q_{0} \varphi_{0}+Q_{2} \varphi_{2}}\left|\psi_{\text {top }}\right|^{2}
$$

This formula has a natural interpretation from the point of view of the wave function interpretation of $\psi_{\text {top }}$ developed in $[13,14]$ as an interpretation of the holomorphic anomaly $[15,16]$. Namely, equation $(2.5)$ expresses $\Omega$ as the "Wigner function" (phase-space density) associated to $\psi_{\text {top. }}$. The background-independent generalization of this transform and its relation to the counting of black hole states has been further elucidated in ref. [17].

The formula (2.5) also illustrates a crucial point about the conjecture: in order to use it to compute $\Omega$, one would need to know the full $\left|\psi_{\text {top }}\right|^{2}$, not only its asymptotic expansion for $g_{\text {top }} \ll 1$. Put another way, knowing the BPS degeneracies $\Omega$ is in some sense equivalent to having a non-perturbative completion of $\left|\psi_{\text {top }}\right|^{2}$.

\subsection{A solvable example}

In this section, we review the work of refs. $[5,6]$ which argued that the conjecture (2.2) holds in the case where $X$ is a particular non-compact CalabiYau three-fold, namely the total space of a holomorphic vector bundle over a compact Riemann surface $\Sigma$ of genus $g$,

$$
X=\mathcal{L}_{-p} \oplus \mathcal{L}_{p+2 g-2} \rightarrow \Sigma,
$$

for some $p>0 .^{2}$

The idea is that for this $X$ one can use two-dimensional Yang-Mills theory to compute $Z_{\mathrm{BH}}$, as follows. Suppose we wrap $N$ D4-branes on the holomorphic 4-cycle

$$
\mathcal{D}=\mathcal{L}_{-p} \rightarrow \Sigma
$$

Then the theory on the D4-branes (in the Calabi-Yau directions) is the $\mathcal{N}=4$ supersymmetric Yang-Mills theory, or more precisely a topologically twisted version of that theory, as explained in ref. [18]. The path integral in this theory includes configurations in which D0-branes, and D2-branes wrapping $\Sigma$, are bound to the D4-branes. Hence the partition function of

\footnotetext{
${ }^{2}$ By $\mathcal{L}_{k}$ we mean a holomorphic line bundle of degree $k$ over $\Sigma$.
} 
the four-dimensional twisted supersymmetric gauge theory computes a sum over the mixed ensemble of BPS states which we considered above. The D4 and D6-brane charges are:

$$
\begin{aligned}
Q_{4} & =N[\mathcal{D}], \\
Q_{6} & =0 .
\end{aligned}
$$

The chemical potentials for the brane charges are roughly given by the masses of the branes (for the D2-branes we turn on a Ramond-Ramond field $\theta$ ):

$$
\begin{aligned}
& \varphi_{0}=4 \pi^{2} / g_{s}, \\
& \varphi_{2}=2 \pi p \theta / g_{s}
\end{aligned}
$$

Since the gauge theory sums over all brane charges we can now write ${ }^{3}$

$$
Z_{\mathrm{YM}}=Z_{\mathrm{BH}}
$$

It was argued in ref. [5] that, for the purpose of computing $Z_{\mathrm{YM}}$, we can restrict to field configurations in the $\mathcal{N}=4$ theory which are invariant under the $U(1)$ action on the fibers of $\mathcal{L}_{-p}$. One then obtains $Z_{\mathrm{YM}}$ as the partition function of a $q$-deformed Yang-Mills theory on $\Sigma$ (see Appendix B), where $\Sigma$ has area $p$ and the parameters are fixed by

$$
\theta_{\mathrm{YM}}=\theta, \quad g_{\mathrm{YM}}^{2}=g_{s}, \quad q=\mathrm{e}^{-g_{s}} .
$$

The $q$-deformed Yang-Mills theory is a relative of the ordinary Yang-Mills theory in two dimensions, and shares with that theory the property of being exactly solvable; the topological string on $X$ is also exactly solvable to all orders in perturbation theory (using recent results of ref. [19] in the case $g>1$ ). Hence we can use $X$ as a testing ground for (2.2). More precisely, since we do not have a good understanding of the non-perturbative topological string, what we can do is look at the asymptotic expansion of $\left|\psi_{\text {top }}\right|^{2}$ in the limit $g_{s} \ll 1$, with $t$ fixed. On the physical side this corresponds to taking $\varphi_{0}, \varphi_{2}$, and $N$ to infinity with fixed ratios (this is a 't Hooft limit in the Yang-Mills theory).

In this limit one finds that $Z_{\mathrm{YM}}$ factorizes into a sum of "conformal blocks," each given by the topological string on $X$, with some D-branes

\footnotetext{
${ }^{3}$ There are some subtleties because of the non-compactness of $X$, as noted in ref. [6]: $Z_{\mathrm{YM}}$ turns out to give a sum over finitely many sectors, each with a $g_{s}$-dependent prefactor.
} 
inserted as we will explain below:

$$
\begin{aligned}
Z_{\mathrm{YM}}\left(\varphi_{0}, \varphi_{2}, N\right)= & \sum_{R_{1}^{\prime}, \ldots, R_{|2 g-2|}^{\prime}} \sum_{l \in \mathbb{Z}} \psi_{\mathrm{top}}^{R_{1}^{\prime}, \ldots, R_{|2 g-2|}^{\prime}}\left(g_{\mathrm{top}}, t+l p g_{\mathrm{top}}\right) \\
& \times \frac{\psi_{\mathrm{top}}^{R_{1}^{\prime}, \ldots, R_{|2 g-2|}^{\prime}}\left(g_{\mathrm{top}}, t-l p g_{\mathrm{top}}\right)}{}+\mathcal{O}\left(\mathrm{e}^{-N}\right) .
\end{aligned}
$$

Here $t$ and $g_{\text {top }}$ are as dictated by (2.3) and (2.4), namely,

$$
\begin{aligned}
g_{\text {top }} & =4 \pi^{2} / \varphi_{0}=g_{s}, \\
t & =\frac{1}{2} g_{\text {top }}\left(\#(\Sigma \cap \mathcal{D}) N+i \varphi_{2} / \pi\right)=\frac{1}{2} N(p+2 g-2) g_{s}+i p \theta .
\end{aligned}
$$

The index $l$ was interpreted in ref. [5] as measuring the Ramond-Ramond flux through $\Sigma$. The labels $R_{i}^{\prime}$ are subtler; they appear only when $g \neq 1$, in which case they were interpreted in ref. [6] as running over boundary conditions on $|2 g-2|$ infinite stacks of D-branes (which we christen "ghost" D-branes) in the topological string. Each stack lies on a Lagrangian submanifold of $X$, intersecting $\mathcal{D}$ in an $S^{1}$ in the fiber of $\mathcal{L}_{p+2 g-2}$ over a point. The boundary conditions on each stack are specified by a choice of a Young diagram $R^{\prime}{ }^{4}$

The form of (2.14) looks different from that of (2.2). Nevertheless, as we will explain in the following section, the sum over Young diagrams $R_{i}^{\prime}$ is indeed consistent with (2.2), when we take into account extra closed string moduli at infinity.

\section{Revisiting the closed string theory}

In this section, we revisit the relation between 2-d Yang-Mills theory and the closed topological string, with the aim of giving a better interpretation to the sum over chiral blocks and the appearance of "ghost" D-branes.

As we reviewed in Section 2, the partition function of the twisted $U(N)$ Yang-Mills theory on $\mathcal{D}=\mathcal{L}_{-p} \rightarrow \Sigma$ factorizes at large $N$ as a sum of blocks, each of which can be interpreted as the square of a topological string amplitude involving $2 g-2$ infinite stacks of ghost branes. Introducing a $U(\infty)$ valued holonomy $U_{i}^{\prime}=e^{u_{i}^{\prime}}$ on each stack of ghost branes, we can rewrite

\footnotetext{
${ }^{4}$ All primed quantities which appear in this article are associated to these ghost D-branes.
} 
$(2.14)$ as

$$
\begin{aligned}
Z_{\mathrm{YM}}= & \sum_{l \in \mathbb{Z}} \int d_{\mathrm{H}} u_{1}^{\prime} \cdots d_{\mathrm{H}} u_{2 g-2}^{\prime} \psi_{\mathrm{top}}^{\mathrm{g}}\left(g_{\mathrm{top}}, u^{\prime}, t+l p g_{\mathrm{top}}\right) \\
& \times \overline{\psi_{\mathrm{top}}^{\mathrm{g}}\left(g_{\mathrm{top}}, u^{\prime}, t-l p g_{\mathrm{top}}\right)}
\end{aligned}
$$

where

$$
\psi_{\text {top }}^{\mathrm{g}}\left(g_{\mathrm{top}}, u^{\prime}, t\right)=\sum_{R_{1}^{\prime}, \ldots R_{2 g-2}^{\prime}} \psi_{\text {top }}^{R_{1}^{\prime}, \ldots, R_{2 g-2}^{\prime}}\left(g_{\mathrm{top}}, t\right) \mathrm{e}^{-\frac{1}{2} N g_{s} \sum_{i=1}^{2 g-2}\left|R_{i}^{\prime}\right|} \prod_{i=1}^{2 g-2} s_{R_{i}^{\prime}}\left(\mathrm{e}^{u_{i}^{\prime}}\right) .
$$

For $g=0$ the formula is similar, except that the role of ghost branes and ghost antibranes are reversed in the antitopological amplitude:

$$
Z_{\mathrm{YM}}=\sum_{l \in \mathbb{Z}} \int d_{\mathrm{H}} u_{1}^{\prime} d_{\mathrm{H}} u_{2}^{\prime} \psi_{\mathrm{top}}^{\mathrm{g}}\left(g_{\mathrm{top}}, u^{\prime}, t+l p g_{\mathrm{top}}\right) \overline{\psi_{\mathrm{top}}^{\mathrm{a}}\left(g_{\mathrm{top}}, u^{\prime}, t-l p g_{\mathrm{top}}\right)},
$$

where

$$
\begin{aligned}
\psi_{\text {top }}^{\mathrm{g}}\left(g_{\mathrm{top}}, u^{\prime}, t\right)= & \sum_{R_{1}^{\prime}, R_{2}^{\prime}} \psi_{\text {top }}^{R_{1}^{\prime}, R_{2}^{\prime}}\left(g_{\mathrm{top}}, t\right) \mathrm{e}^{-\frac{1}{2} N g_{s}\left(\left|R_{1}^{\prime}\right|+\left|R_{2}^{\prime}\right|\right)} s_{R_{1}^{\prime}}\left(\mathrm{e}^{u_{1}^{\prime}}\right) s_{R_{2}^{\prime}}\left(\mathrm{e}^{u_{2}^{\prime}}\right), \\
\psi_{\text {top }}^{\mathrm{a}}\left(g_{\mathrm{top}}, u^{\prime}, t\right)= & \sum_{R_{1}^{\prime}, R_{2}^{\prime}}(-)^{\left|R_{1}^{\prime}\right|+\left|R_{2}^{\prime}\right|} \psi_{\text {top }}^{R_{1}^{\prime}, R_{2}^{\prime}}\left(g_{\mathrm{top}}, t\right) \mathrm{e}^{-\frac{1}{2} N g_{s}\left(\left|R_{1}^{\prime}\right|+\left|R_{2}^{\prime}\right|\right)} s_{R_{1}^{\prime t}} \\
& \times\left(\mathrm{e}^{u_{1}^{\prime}}\right) s_{R_{2}^{\prime t}}\left(\mathrm{e}^{u_{2}^{\prime}}\right) .
\end{aligned}
$$

The change from branes to antibranes is reflected in the signs $(-)^{\left|R^{\prime}\right|}$ and the switch $R^{\prime} \rightarrow R^{\prime t}$ between $\psi^{\mathrm{g}}$ and $\psi^{\mathrm{a}}$, as in ref. [20].

Now note that (3.1) and (3.3) look like the integral (2.5), which computes the microcanonical degeneracies by integrating over the imaginary part of each Kähler modulus whereas the real part is fixed by the corresponding magnetic charge. Indeed, the factor $\mathrm{e}^{-\frac{1}{2} N g_{s} \sum_{i=1}^{2 g-2}\left|R_{i}^{\prime}\right|}$ could be absorbed in $U^{\prime}$, at the expense of making it non-unitary: this just amounts to giving $u^{\prime}$ a real part. This is reminiscent of the "attractor" formula (2.4), which says the real part of the Kähler modulus is related to the charge. So indeed, equation (3.1) could be consistent with the conjecture (2.2), if we somehow regard $u^{\prime}$ as an extra closed string modulus; then there would be electric and magnetic charges corresponding to it, and (3.1) says that $Z_{\mathrm{YM}}$ is the partition function of an ensemble in which we have fixed these charges. As we will now explain, this interpretation of $u^{\prime}$ is indeed plausible. 


\subsection{Open vs. closed}

We explained above that the non-perturbative completion of the closed topological string appears to involve Lagrangian D-branes on the CalabiYau manifold. The appearance of open string amplitudes in this context is surprising, since in the physical string this would have half as much supersymmetry as we have available. As we will now argue, the correct interpretation involves not open but closed strings.

Namely, as was shown in ref. [21], in the topological string, inserting non-compact D-branes is equivalent to turning on certain non-normalizable deformations of the Calabi-Yau. This is an open-closed duality of the topological string, generalizing the well-known duality for D-branes on compact cycles. This means that, at the level of the topological string, we can interpret the modulus $U^{\prime}$ in (3.1) as either corresponding to an open string configuration or to a boundary condition at infinity of the closed topological string. In the physical string theory, however, we do not have this freedom; since there are no Ramond-Ramond fluxes turned on, the only interpretation available is the closed string one.

The torus symmetries of the Calabi-Yau manifold can be used to constrain the types of deformation that we consider. Namely, the Lagrangian D-branes to which (3.2) corresponds respect the torus symmetries, and the gravitational backreaction they create does so as well. Such torus invariant deformations, normalizable and not, were studied in ref. [21], so we can borrow the results of that paper. The topological string theory in ref. [21] was described as the theory of a chiral boson on a Riemann surface, and the Lagrangian D-branes were coherent states of this chiral boson. (Note here that we are using the mirror B-model language. The global action of mirror symmetry on $X$ is not relevant for us; this is merely a convenient language in which to describe the behavior near an asymptotic infinity). The nonnormalizable deformations of the Calabi-Yau near an asymptotic infinity ${ }^{5}$ can be parameterized by the coherent states of the chiral boson:

$$
|\tau\rangle=\exp \left(\sum_{n>0} \tau_{n} \alpha_{-n}\right)|0\rangle,
$$

where $\alpha_{n}$ are the chiral boson creation and annihilation operators.

\footnotetext{
${ }^{5}$ In the cases studied in ref. [21] there is a clear notion of what "an asymptotic infinity" means: it means a toric 2-cycle which extends to infinity. In the cases, we are considering here the situation is not as rigorously understood, but we will make some comments below.
} 
The parameters $\tau$ are related to the D-brane holonomies by

$$
\tau_{n}=g_{s} \operatorname{Tr} U^{\prime n}
$$

where $\operatorname{Tr}$ denotes the trace in the fundamental representation. The factor of $g_{s}$ is needed to convert an open string amplitude in terms of $U$ to a closed string amplitude in terms of $t$; it appears because a trace of $U$ in the fundamental representation couples to a hole in the string worldsheet, and the hole is in turn weighted by $g_{s}$ in the string perturbation expansion. In this sense, the open string modulus $U$ can be traded for the infinite collection of closed string moduli $\tau_{n}$.

Actually, it is more convenient to reparameterize slightly by taking a logarithm, writing $\tau_{n}=\mathrm{e}^{-t_{n}}$. The point is that the A model partition function turns out to be an expansion in $\mathrm{e}^{-t_{n}}$, so the moduli $t_{n}$ appear on the same footing as the Kähler volumes $t$ of compact cycles. Indeed, we can think of them as representing Kähler volumes of classes in $H_{2}(X, \mathbb{Z})$ (with some appropriate notion of what $H_{2}(X, \mathbb{Z})$ means for this non-compact $\left.X\right)$. What can we say about these classes? In the cases considered in ref. [21], for each asymptotic infinity there is a holomorphic $\operatorname{disc} C$ which "ends" on it, and $t_{n}$ represents a class which contains $n[C]$ as well as some extra contributions at infinity. In the open string language, the disc $C$ can be thought of as ending on the Lagrangian branes which represent the deformations at this asymptotic infinity.

\subsection{The attractor mechanism and ghost D-branes}

Now we come to the interpretation of the shift $U^{\prime} \rightarrow U^{\prime} \mathrm{e}^{-\frac{1}{2} N g_{s}}$, or equivalently

$$
\operatorname{Re} t_{n}=\frac{1}{2} n N g_{s}
$$

Such shifts have frequently appeared in the topological string in the presence of D-branes. Here, we can understand the shift as a reflection of the attractor mechanism on the closed string moduli. Namely, in the case we are considering here, $C$ is a disc in the fiber of $\mathcal{L}_{p+2 g-2}$, which intersects $\mathcal{D}$ at one point, as shown in figure 1 . Then $\frac{1}{2} n N g_{s}$ is exactly the expected attractor value for the Kähler modulus $t_{n}$, as follows from (2.4), the fact that the D4-brane charge is $Q_{4}=N[\mathcal{D}]$, and $\#(C \cap \mathcal{D})=1$. (Whatever the extra contributions at infinity to the class represented by $t_{n}$ are, they have zero intersection number with $\mathcal{D}$, so they do not affect the attractor modulus). 


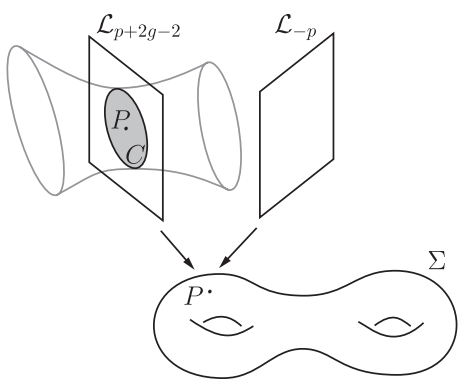

Figure 1: The disc $C$ in the fiber of $\mathcal{L}_{p+2 g-2}$ over a point $P$ on the Riemann surface $\Sigma ; C$ meets $\mathcal{D}$ only at $P$, and the boundary of $C$ lies on the Lagrangian submanifold representing this asymptotic infinity.

\subsection{Why $2 g-2$ asymptotic infinities}

The discussion of the last few sections raises a natural question: why are there precisely $|2 g-2|$ asymptotic infinities on $X$ where we can have deformations?

In general, we should have expected that in a non-compact Calabi-Yau we should include some closed string moduli coming from infinity. However, in problems with symmetries, it is natural to conjecture that the only relevant extra moduli from infinity are invariant under the corresponding symmetries. We will assume this here and look for symmetries in our problem which simplify the task of specifying the closed string moduli coming from infinity.

A priori, one might have expected boundary moduli associated to the $\mathbb{C}^{2}$ fiber over each point of the Riemann surface. Here we have in addition D4-branes wrapping a line bundle over the Riemann surface. We claim that this implies that effectively we should view that direction as "compact," or more precisely, we should view it as a degenerate limit of a compact 4-cycle. After this reduction, we would expect to find boundary moduli corresponding to a $\mathbb{C}$ fiber over each point on the Riemann surface.

However, there are symmetries of the problem coming from meromorphic vector fields on the Riemann surface. Hence the variation of the data at infinity can be localized at poles or zeroes of such a vector field (deleting these points would give a well-defined free action). A generic holomorphic vector field on a Riemann surface of genus $g>1$ is non-vanishing and well defined away from $2 g-2$ poles, which we identify with places where the asymptotic boundary condition at infinity can be localized. The local picture is as shown in figure 2 . 


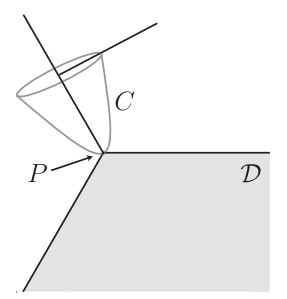

Figure 2: A rough toric representation of the behavior of $X$ in a neighborhood of a singularity of the vector field $v$ described in the text. Two of the three $U(1)$ actions making up the toric fiber are the rotations of the line bundles $\mathcal{L}_{-p} \oplus \mathcal{L}_{p+2 g-2}$ and the third is the action of $v$. The toric base of the divisor $\mathcal{D}$ on which the D4-branes are wrapped is indicated, as is the base of the Lagrangian submanifold representing the asymptotic infinity. The disc $C$ ends on this Lagrangian submanifold, meeting $\mathcal{D}$ at the single point $P$.

So the closed string moduli at these $2 g-2$ asymptotic infinities may be identified with the "ghost D-brane" contributions, as discussed above. In the case of genus 1 there are no fixed points, which is consistent with the fact that no ghost D-branes were needed in this case. For genus 0 we have a holomorphic vector field with 2 zeroes, which again suggests that we can localize the contribution from infinity at 2 points.

This is a heuristic argument, but we feel that it captures the correct physics.

\section{The quantum mechanics of open strings}

In Section 2, we reviewed the conjecture of ref. [4] and its relation to the wave function nature of the closed topological string. In this section, we recall the parallel statement for the open topological string. The fact that the open topological string partition function including non-compact branes is a wave function was first noticed in ref. [21], and was crucial in that paper for the solution of the B model. In this section, we will give two ways of understanding this wave function property: a direct route via canonical quantization of Chern-Simons theory; a more indirect one via the holomorphic anomaly (background dependence) for open strings.

\subsection{Canonical quantization in Chern-Simons}

Recall that the topological A model string theory on $M$ D-branes wrapped on a Lagrangian cycle $L$ is the $U(M)$ Chern-Simons theory deformed by 
worldsheet instanton corrections:

$$
S=S_{\mathrm{CS}}+S_{\mathrm{inst}},
$$

where

$$
S_{\mathrm{CS}}=\frac{4 \pi i}{k} \int_{L} \operatorname{Tr}\left(A \wedge d A+\frac{2}{3} A \wedge A \wedge A\right),
$$

and $S_{\text {inst }}$ is the contribution from worldsheet instantons with boundaries on $L$. If $L$ is non-compact, then we should consider it as having a boundary $\partial L$ at infinity; the path integral on $L$ then gives a wave function in the Hilbert space of Chern-Simons on the boundary. The case of interest for the rest of this article is $L \simeq \mathbb{R}^{2} \times S^{1}$, which has $\partial L=T^{2}$; from now on we specialize to that case, although the discussion could be made more general.

To find which state the topological open string theory picks, we need to recall some facts about canonical quantization of the $U(M)$ Chern-Simons theory on $T^{2} \times \mathbb{R}$, viewing $\mathbb{R}$ as the "time" direction. We will be brief here; see e.g. [22] for more details. Integrating over the time component of the gauge field localizes the path integral to flat connections on $T^{2}$ :

$$
\int \mathcal{D} A^{\prime} \delta\left(F^{\prime}\right) \exp \left(\frac{2 \pi i}{k} \int_{T^{2} \times R} \operatorname{Tr} A^{\prime} \partial_{t} A^{\prime} d t\right) .
$$

Above $A^{\prime}$ is a connection on $T^{2}$, which we can write (up to conjugation) as

$$
A^{\prime}=u d \theta_{u}+v d \theta_{v}
$$

where $u$ and $v$ are the components of $A^{\prime}$ along two linearly independent cycles of $T^{2}$, with intersection number 1 . From the action (4.3) we see that $u$ and $v$ are conjugate variables: upon quantization we thus expect

$$
[u, v]=i g_{\mathrm{top}}
$$

where $g_{\text {top }}=\frac{2 \pi}{k+M}$. The familiar shift of $k$ by $M$ can be seen by carefully integrating over massive modes [22].

Since $u$ and $v$ are conjugate variables, in computing the Chern-Simons path integral on a manifold with $T^{2}$ boundary, we should fix either $u$ or $v$ on the boundary, but not both, and the wave function will depend on the variable we have chosen to fix. More generally, we could consider a mixed boundary condition where we fix $v+\bar{\tau} u$ where $\bar{\tau}$ is some parameter (the motivation for this notation will become clear later).

Note that in the present context $L \simeq \mathbb{R}^{2} \times S^{1}$ is a solid torus, so there is a unique 1-cycle $\eta \in H_{1}\left(T^{2}, \mathbb{Z}\right)$ which collapses in the interior of $L$. There is thus a canonical choice of polarization for the wave function; namely, one can express it in terms of the holonomy around $\eta$, which we call $v$. In the following section, we will relate this choice to the background dependence ("holomorphic anomaly") of the open topological string. We could have 
tried to choose the "cycle that survives" in the interior of $L$ (corresponding to the holonomy $u$ ), but this is ambiguous up to the shift $u \mapsto u+n v$. This ambiguity will be related to the framing ambiguity of the open topological string.

It can be shown $[22,23]$ that the Chern-Simons path integral on the solid torus, without any insertions and with $u$ fixed on the boundary, is given simply by

$$
\psi_{\text {top }}^{\text {open }}(u)=\langle L \mid u\rangle=1
$$

In the present context, the Chern-Simons action is deformed by worldsheet instantons wrapping holomorphic curves with boundaries on $L$ [24]. Their contribution to $S$ is given by the free energy of the gas of topological open strings:

$$
S_{\text {inst }}(u)=i F_{\text {top }}^{\text {open }}(u) .
$$

We now want to compute the path integral on $L$ with the operator insertion

$$
\exp S_{\text {inst }}(u) \text {. }
$$

Since we are working in the basis of eigenstates of $u$, the insertion just acts by multiplication:

$$
\psi_{\text {top }}^{\text {open }}(u)=\left\langle L\left|\mathrm{e}^{S_{\mathrm{inst}}(u)}\right| u\right\rangle=\mathrm{e}^{i F_{\mathrm{top}}^{\text {open }}(u)}\langle L \mid u\rangle=\mathrm{e}^{i F_{\mathrm{top}}^{\text {open }}(u)} .
$$

So we have identified the topological string partition function $\mathrm{e}^{i F_{\mathrm{top}}^{\mathrm{open}}(u)}$ with a wave function.

Although $v$ is the canonical choice, we will sometimes find it natural to write the wave function in terms of one of the holonomies $u+n v$ instead. The relation between different choices of variable in which to write the wave function is given by a Fourier transform: for example, to transform from $u$ to $v$, one has

$$
\psi_{\text {top }}^{\text {open }}(v)=\int d_{H} u \mathrm{e}^{\frac{i}{g_{\text {top }}} \operatorname{Tr} u v} \psi_{\text {top }}^{\text {open }}(u)
$$

where $d_{\mathrm{H}} u$ is the measure induced from the Haar measure on $U(M)$.

The freedom to choose a variable is crucial because there are some cases in which the Lagrangian cycle $L$ can make a "flop transition." From the perspective of the boundary $\partial L=T^{2}$ nothing special happens at the transition, but in the interior of $L$ the topology changes and in particular the cycle that collapses in the interior is different after the transition. An example of this phenomenon can be seen when $X$ is a toric Calabi-Yau manifold. Moreover, in that case one can use the mirror B model to see that worldsheet instanton corrections eliminate the sharp transition: the different phases are smoothly connected. Thus, in the B model language there is a continuous change of variables which takes us from one choice of holonomy to another. This is 
related to the background dependence of open topological string amplitudes, to which we now turn.

\subsection{Background dependence for the open topological string}

In this section, we take a brief detour to explain the background dependence of the open string topological string. ${ }^{6}$ It was conjectured in ref. [21] that the open topological string partition function depends on a choice of "background" moduli, or equivalently, depends on the antiholomorphic coordinates of the moduli as well as the holomorphic ones. This conjecture was advanced in order to explain the fact that the open topological string behaves like a wave function, by analogy to what is known for the closed string case [13]. In the case considered in ref. [21], the geometry of the Calabi-Yau is given (in the mirror B model) by a hypersurface in $\mathbb{C}^{4}$,

$$
F(u, v)-x y=0,
$$

and the mirror of the Lagrangian brane is a brane on a holomorphic curve, specified by the condition $x=0$ together with fixed choices of $u, v$ satisfying $F(u, v)=0$. As noted in refs. [21,23] the geometry with this D-brane included can be viewed as a special (degenerate) limit of a closed string geometry, with the D-brane serving as a source for the holomorphic 3-form; this source changes the usual equation $d \Omega=0$ to

$$
d \Omega=g_{\mathrm{top}} \delta(D)
$$

where $\delta(D)$ denotes a delta function at the locus of the D-brane. We have already used this correspondence in Section 3, where we discussed how the "ghost branes" can be viewed as closed string moduli. Similarly, we can use it to interpret the holomorphic anomaly of closed strings as inducing a holomorphic anomaly (or equivalently a background dependence) for the open string partition function. Here we view the modulus of the open string, given by the choice of $(u, v)$ on the surface $F(u, v)=0$, as a closed string modulus. In fact, borrowing the closed string technology for background dependence developed in refs. $[13,14]$, we immediately deduce that for a given background $\left(u_{0}, v_{0}\right)$ the natural variable for the open string wave function is

$$
v+\bar{\tau} u,
$$

\footnotetext{
${ }^{6}$ This was the original motivation for the present article!
} 
where

$$
\tau=-\left.\frac{\partial v}{\partial u}\right|_{\left(u_{0}, v_{0}\right)} .
$$

Here, we are considering $v$ as a function of $u$ through the implicit relation $F(u, v)=0$, so $\tau$ is the slope of the tangent plane to the Riemann surface at $\left(u_{0}, v_{0}\right)$. Note that $\tau=\partial^{2} F / \partial u^{2}$.

The form (4.13) of the natural variable can be connected to our earlier discussion of the wave function nature of the Chern-Simons theory embedded in the open string; there too we claimed that there is a natural variable for the wave function, namely the holonomy around the cycle of $T^{2}$ which shrinks in the interior of the solid torus. In that classical picture (which neglects the effect of worldsheet instantons), the holonomy around the vanishing cycle is simply $v$; and choosing the background point near an asymptotic infinity of the quantum moduli space, where the classical picture becomes exact, one indeed gets $\tau \rightarrow 0$, so $v+\bar{\tau} u \rightarrow v$. More invariantly, the value of $\tau$ near an asymptotic infinity of the B model Riemann surface approaches the slope of the corresponding line in the A model toric diagram, and this slope indeed determines the collapsing cycle of the toric fiber.

Note that in order to go off the real locus $\tau=\bar{\tau}$ we need to recall that the Chern-Simons holonomies are complexified in the context of topological strings (to include the moduli which move the brane); in the geometric motivation we gave before we had essentially turned those off. It would be interesting to understand this relation off the real locus.

\section{The open string conjecture}

As we reviewed in Section 2, the closed topological string wave function on a Calabi-Yau space $X$ is believed to compute the large-charge asymptotics of an index which counts BPS states in four dimensions, and this index has an interpretation as the Wigner function of $\psi_{\text {top }}$. On the other hand, we just saw in Section 4 that the open topological string partition function $\psi_{\text {top }}^{\text {open }}$ with non-compact D-branes is also naturally considered as a wave function. So we could construct a Wigner function from this wave function, and then a natural question is whether this Wigner function also has an interpretation as counting BPS states. We will argue that it does.

We embed the open topological string in the superstring in a familiar way [25]. Namely, consider D4-branes wrapping a special Lagrangian cycle $L \subset$ $X$. Then there are open D2-branes ending on these D4-branes. These give rise to BPS particles in the two-dimensional supersymmetric gauge theory on 
the non-compact directions of the D4-branes; we will interpret the charge $\mathcal{Q}_{\text {e }}$ as counting these BPS particles. The gauge theory in question also supports BPS domain walls; we will interpret $\mathcal{Q}_{\mathrm{m}}$ as measuring the domain wall charge.

Altogether then, we will conjecture below that the open topological string, on a Calabi-Yau space $X$ with Lagrangian branes included, computes the large-charge asymptotics of an index which counts open D2-branes, and their domain wall counterparts, bound to any number of closed D0, D2, D4 and D6-branes. Furthermore, we will describe one context in which some aspects of this proposal can be checked.

\subsection{Calabi-Yau spaces with branes and BPS particles}

Consider a Calabi-Yau manifold $X$ containing a special Lagrangian 3-cycle $L$. We consider the Type IIA superstring on $X \times \mathbb{R}^{3,1}$, with $M$ D4-branes on $L \times \mathbb{R}^{1,1}$, which we will call the "background branes." For simplicity, we assume $L$ has the topology

$$
L \simeq \mathbb{R}^{2} \times S^{1}
$$

The dimensionally reduced theory on the $\mathbb{R}^{1,1}$ part of the background branes is a $(2,2)$ supersymmetric gauge theory. Its field content can be understood as follows [25]. Since $b_{1}(L)=1$ it follows [26] that $L$ has one real modulus $r$; this modulus pairs up with the Wilson line of the worldvolume gauge field $\oint A$ to give a complex field

$$
u=r+i \oint A
$$

One also gets a gauge field in $\mathbb{R}^{1,1}$ by integrating the world-volume two-form $B$ (which is the magnetic dual to the gauge field $A$ on the D4-brane, defined by $d * A=d B$ ) over the $S^{1}$ of $L$. Since there are $M$ D4-branes, the theory has (at least) a magnetic $U(1)^{M}$ gauge symmetry. The field $u$ should be viewed as the lowest component of a twisted chiral multiplet, whose top component is the field strength of the magnetic gauge field in two dimensions.

There is an obvious way of getting BPS particles in this theory. Suppose for a moment that $M=1$ (a single Lagrangian brane). Let $\gamma \in H_{1}(L, \mathbb{Z})$ denote the homology class of the $S^{1}$ in $L$. Since the Calabi-Yau has no 
non-contractible 1-cycles, this $\gamma$ is a boundary in $X$; so there exists some $D$ with

$$
[\partial D]=\gamma
$$

Open D2-branes wrapped on $D$ give rise to particles charged under the $U(1)$ gauge field of the two-dimensional theory; if $D$ is a holomorphic disc, then these particles are BPS.

\subsection{The conjecture}

Now, to motivate our conjecture, recall from Section 2 that in the closed string case (without the background branes) we have the relation

$$
Z_{\mathrm{BH}}\left(Q_{6}, Q_{4}, \varphi_{2}, \varphi_{0}\right)=\sum_{Q_{0}, Q_{2}} \Omega_{Q_{6}, Q_{4}, Q_{2}, Q_{0}} \mathrm{e}^{-Q_{2} \varphi_{2}-Q_{0} \varphi_{0}}=\left|\psi_{\text {top }}\left(g_{\text {top }}, t\right)\right|^{2},
$$

where $\varphi_{2}=\operatorname{Im} 2 \pi t / g_{\text {top }}$ and $\varphi_{0}=\operatorname{Im~} 4 \pi^{2} / g_{\text {top }}$ as given in (2.3) and (2.4). We wish to generalize this conjecture to the open topological string. What is the appropriate ensemble to consider? Since the closed D2-branes are "light electric states" in the closed string ensemble, which we sum over with chemical potentials, it is natural to try treating the open D2-branes in the same way. Thus, in formulating our conjecture we consider these BPS states as "electric charges," and sum over them with a chemical potential $\varphi_{\mathrm{e}}^{\text {open }}$. We also expect to have a "magnetic charge," which we fix to the some value $\mathcal{Q}_{\mathrm{m}}^{\text {open }}$; we will discuss these charges further below. ${ }^{7}$ The partition function of the ensemble thus obtained is a simple generalization of (5.4),

$$
\begin{aligned}
Z_{\mathrm{BPS}}^{\text {open }}\left(Q_{6}, Q_{4}, \mathcal{Q}_{\mathrm{m}}^{\text {open }}, \varphi_{2}, \varphi_{0}, \varphi_{\mathrm{e}}^{\text {open }}\right)= & \sum_{Q_{0}, Q_{2}, \mathcal{Q}_{\mathrm{e}}^{\text {open }}} \Omega_{Q_{6}, Q_{4}, Q_{2}, Q_{0}, \mathcal{Q}_{\mathrm{e}}^{\text {open }}, \mathcal{Q}_{\mathrm{m}}^{\text {open }}} \\
& \times \mathrm{e}^{-Q_{2} \varphi_{2}-Q_{0} \varphi_{0}-\mathcal{Q}_{\mathrm{e}}^{\text {open }} \varphi_{\mathrm{e}}^{\text {open }}} .
\end{aligned}
$$

We conjecture that the relation of $Z_{\mathrm{BPS}}^{\text {open }}$ to the topological string is a direct generalization of (5.4),

$$
Z_{\mathrm{BPS}}^{\mathrm{open}}\left(Q_{6}, Q_{4}, \mathcal{Q}_{\mathrm{m}}^{\mathrm{open}}, \varphi_{2}, \varphi_{0}, \varphi_{\mathrm{e}}^{\mathrm{open}}\right)=\left|\psi_{\text {top }}^{\text {open }}\left(g_{\mathrm{top}}, t, u\right)\right|^{2},
$$

where $\psi_{\text {top }}^{\text {open }}$ is the topological A model partition function on $X$, including open strings ending on $M$ D-branes on $L$ as well as closed strings.

In this conjecture, the closed string moduli $g_{\text {top }}, t$ are determined by the attractor mechanism as before. What about the open string modulus $u$ ? The formula $\varphi_{2}=\operatorname{Im} 2 \pi t / g_{\text {top }}$ for the closed D2-brane chemical potential

\footnotetext{
"The terminology "electric" and "magnetic" here is chosen by analogy to the closed string case. The charges we are discussing here are both associated to point particles, which are not electric-magnetic duals in the theory on $\mathbb{R}^{1,1}$.
} 
suggests that the open D2-brane chemical potential should be related to $u$ by

$$
\varphi_{\mathrm{e}}^{\mathrm{open}}=\operatorname{Im} 2 \pi u / g_{\mathrm{top}}
$$

We will verify this identification of $\operatorname{Im} u$ in an explicit example below. The real part of $u$ should be fixed by the charge $\mathcal{Q}_{\mathrm{m}}^{\text {open }}$, as we now discuss.

\subsection{Adding magnetic charges}

What is the spacetime meaning of the "magnetic" charge $\mathcal{Q}_{\mathrm{m}}^{\text {open }}$, and its relation to the real part of the modulus $u$ ? We can make a plausible guess by exploiting the symmetry between $u$ and its conjugate $v$. Namely, as noted in Section 4 , it is possible for $L$ to undergo a flop transition to a new phase parameterized by a different parameter $v$ (representing the holonomy of the gauge field around a new $S^{1}$ which was contractible in the old phase). The two phases are smoothly connected in the quantum topological string theory and also in the physical one, but they correspond to different classical descriptions of the physics. The most economical assumption would then be that the excitations which we are calling "electric" in one description are the same as the ones which we are calling "magnetic" in the other. In this section we explore the consequences of this assumption (without being too careful about the factors of $i$ which appear). We discuss only the open string sector, suppressing the closed strings, and drop the label "open" from our notation for simplicity.

First, we can write down the precise form of $u$, using the fact that $\psi_{\text {top }}(v)$ is related to $\psi_{\text {top }}(u)$ by the Fourier transform (4.10), or equivalently

$$
[u, v]=i g_{\mathrm{top}} .
$$

The dictionary between our statistical ensemble and the quantummechanical picture requires the relations

$$
\left[\mathcal{Q}_{\mathrm{e}}, \varphi_{\mathrm{e}}\right]=1=\left[\mathcal{Q}_{\mathrm{m}}, \varphi_{\mathrm{m}}\right]
$$

since we cannot fix the charges and the chemical potentials at the same time. On the other hand, we can fix the charges simultaneously, so

$$
\left[\mathcal{Q}_{\mathrm{e}}, \mathcal{Q}_{\mathrm{m}}\right]=0=\left[\varphi_{\mathrm{e}}, \varphi_{\mathrm{m}}\right]
$$

The consistency of (5.8)-(5.10) with $\operatorname{Im} u=g_{\mathrm{top}} \varphi_{\mathrm{e}} / 2 \pi$ then requires

$$
\operatorname{Re} u=\pi \mathcal{Q}_{\mathrm{m}}, \quad \operatorname{Re} v=\pi \mathcal{Q}_{\mathrm{e}} .
$$

Equation (5.11) completes our conjecture (5.6), except that we have not been precise about how to fix the zero of Re $u$ or $\operatorname{Re} v$. We do not have a general proposal for how this should be done, although we will see how it works in an example below. 
Note that the expectation value of $v$ in the state corresponding to the open string wave function $\psi_{\text {top }}(u)=\exp \left(i F_{\text {top }}(u)\right)$ is given by

$$
v=g_{s} \partial_{u} F_{\text {top }}(u)
$$

(the semi-classical version of this equation was discovered in ref. [27]). This is precisely analogous to the special geometry relations of the closed string. In this sense, equation (5.11) seems to describe an open string analog of the attractor mechanism that fixes the moduli to values determined by charges of BPS states. It would be interesting to study this attractor mechanism directly in the physical theory.

Our identification of the parameters leads to two formulas for the Wigner function, i.e. the degeneracies of BPS states,

$$
\begin{aligned}
\Omega_{\mathcal{Q}_{\mathrm{e}}, \mathcal{Q}_{\mathrm{m}}} & =\int d \varphi_{\mathrm{e}} \mathrm{e}^{-\mathcal{Q}_{\mathrm{e}} \varphi_{\mathrm{e}}} \psi\left(u=\frac{i g_{\mathrm{top}}}{2 \pi} \varphi_{\mathrm{e}}+\pi \mathcal{Q}_{\mathrm{m}}\right) \overline{\psi\left(u=\frac{i g_{\mathrm{top}}}{2 \pi} \varphi_{\mathrm{e}}+\pi \mathcal{Q}_{\mathrm{m}}\right)}, \\
& =\int d \varphi_{\mathrm{m}} \mathrm{e}^{-\mathcal{Q}_{\mathrm{m}} \varphi_{\mathrm{m}}} \psi\left(v=\frac{i g_{\mathrm{top}}}{2 \pi} \varphi_{\mathrm{m}}+\pi \mathcal{Q}_{\mathrm{e}}\right) \overline{\psi\left(v=\frac{i g_{\mathrm{top}}}{2 \pi} \varphi_{\mathrm{m}}+\pi \mathcal{Q}_{\mathrm{m}}\right)} .
\end{aligned}
$$

(The arguments we gave above about commutation relations are equivalent to the statement that these two formulas are indeed related by Fourier transforming $\psi(u) \leftrightarrow \psi(v))$. Put another way, $\psi(u)$ and $\psi(v)$ sum over conjugate ensembles:

$$
\begin{aligned}
& \left|\psi\left(u=\frac{i g_{\mathrm{top}}}{2 \pi} \varphi_{\mathrm{e}}+\pi \mathcal{Q}_{\mathrm{m}}\right)\right|^{2}=\sum_{\mathcal{Q}_{\mathrm{e}}} \Omega_{\mathcal{Q}_{\mathrm{e}}, \mathcal{Q}_{\mathrm{m}}} \mathrm{e}^{-\mathcal{Q}_{\mathrm{e}} \varphi_{\mathrm{e}}} \\
& \left|\psi\left(v=\frac{i g_{\mathrm{top}}}{2 \pi} \varphi_{\mathrm{m}}+\pi \mathcal{Q}_{\mathrm{e}}\right)\right|^{2}=\sum_{\mathcal{Q}_{\mathrm{m}}} \Omega_{\mathcal{Q}_{\mathrm{e}}, \mathcal{Q}_{\mathrm{m}}} \mathrm{e}^{-\mathcal{Q}_{\mathrm{m}} \varphi_{\mathrm{m}}}
\end{aligned}
$$

In the above we implicitly chose some framing for the open string wave function $\psi(u)$, and one could ask what is the meaning of changing the framing. As discussed in ref. [21], the effect of shifting the framing by $k$ units is $\psi^{(k)}(u)=\mathrm{e}^{-i k g_{\mathrm{top}} \partial_{u}^{2}} \psi(u)$. From this and (5.15), it follows that $\psi^{(k)}$ sums over an ensemble in which we have a chemical potential for dyons of charge $(1, k)$ :

$$
\left|\psi^{(k)}\left(u=\frac{i g_{\mathrm{top}}}{2 \pi} \varphi_{\mathrm{e}}+\pi \mathcal{Q}_{\mathrm{m}}\right)\right|^{2}=\sum_{\mathcal{Q}_{\mathrm{e}}} \Omega_{\mathcal{Q}_{\mathrm{e}}, \mathcal{Q}_{\mathrm{m}}+k \mathcal{Q}_{\mathrm{e}}} \mathrm{e}^{-\mathcal{Q}_{\mathrm{e}} \varphi_{\mathrm{e}}} .
$$

So far we have discussed the magnetic charge $\mathcal{Q}_{\mathrm{m}}$ abstractly in terms of its relation to the real part of the topological string modulus, but our assumption also leads to a natural description of the meaning of the magnetic 


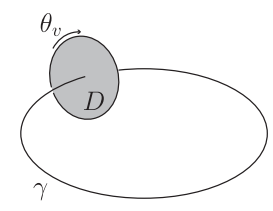

Figure 3: The 1-cycle $\gamma$ and its dual cycle $D$ inside $L$.

charges in the physical theory. To understand this, note first that turning on electric charge $\mathcal{Q}_{\mathrm{e}}$, arising from open D2-branes ending on $\gamma \subset L$, can be equivalently described as turning on magnetic flux on the background D4-brane. This is because the D2-brane ending on $L$ looks like a monopole string from the point of view of the gauge theory on the D4-brane. So, letting $D$ denote any 2-cycle in $L$ dual to $\gamma$ (see figure 3 ), we have

$$
\int_{\mathbb{R} \times D} d F=2 \pi \mathcal{Q}_{\mathrm{e}}
$$

where $\mathbb{R}$ denotes the spatial $x$-direction in $\mathbb{R}^{1,1}$. In particular, we could choose $D$ to be the disc obtained by filling in the 1-cycle $S^{1}$ corresponding to $v$, which opens up after the flop transition. Then (5.18) is equivalent to

$$
\int_{\mathbb{R} \times \partial D} F_{x \theta_{v}} d x d \theta_{v}=2 \pi \mathcal{Q}_{\mathrm{e}}
$$

Alternatively, as $F_{x \theta_{v}}=\partial_{x} A_{\theta_{v}}$ and

$$
\oint_{\partial D} A_{\theta_{v}} d \theta_{v}=\operatorname{Im} v
$$

we see that as we cross the D2-branes in the $x$-direction $v$ jumps by $2 \pi i \mathcal{Q}_{\mathrm{e}}$. Since exchanging electric and magnetic charges corresponds to exchanging $u$ and $v$, it follows that turning on $\mathcal{Q}_{\mathrm{m}}$ units of magnetic charge corresponds to having a domain wall where $u$ jumps by $2 \pi i \mathcal{Q}_{\mathrm{m}}$ in going from $x=-\infty$ to $x=+\infty$. Hence these domain walls are the magnetic charges we were seeking.

\subsection{Multiple Lagrangian branes}

In the above discussion, we have been assuming that we have a single background D4-brane. Let us now return to more general case $M \geq 1$. In this 
case $\mathcal{Q}_{\mathrm{e}, \mathrm{m}}^{\mathrm{open}}$ label representations of $U(M) .{ }^{8}$ By a straightforward generalization of the arguments given above, we see that the attractor values of the eigenvalues of $u$ and $v$ are (generalizing (5.11))

$$
\operatorname{Re} u_{i}=\pi\left(\hat{\mathcal{Q}}_{\mathrm{m}}^{\mathrm{open}}\right)_{i}, \quad \operatorname{Re} v_{j}=\pi\left(\hat{\mathcal{Q}}_{\mathrm{e}}^{\mathrm{open}}\right)_{j} .
$$

Here $\hat{\mathcal{Q}}_{\mathrm{m}, \mathrm{e}}^{\text {open }}$ denote the highest weight vectors of the corresponding representations, shifted by the Weyl vector $\rho$ (see Appendix A). The rest of the discussion generalizes similarly.

\section{A solvable example}

After these general considerations we now return to the example we described in Section 2, where $X$ is a rank 2 holomorphic vector bundle over $\Sigma$, and add background D4-branes on $L \times \mathbb{R}^{1,1}$ to the Type IIA theory. In this section, we want to argue that one can use two-dimensional Yang-Mills theory to compute $Z_{\mathrm{BPS}}^{\mathrm{open}}$, generalizing the discussion of Section 2. Our arguments will be heuristic, but they lead to a definite prescription which is natural and fits in well with our conjectures.

How is the discussion of Section 2 modified by the introduction of the background branes? The $L$ we will consider meet $\mathcal{D}$ along a circle, which we call $\gamma$. Hence in the gauge theory on $\mathcal{D}$ there will be extra massless string states localized along $\gamma$, in the bifundamental of $U(M) \times U(N)$. By condensing these string states (going out along a Higgs branch), i.e. turning on a vacuum expectation value of the form

$$
\left(\begin{array}{ccc}
1 & & \\
& \ddots & \\
& & 1 \\
0 & \cdots & 0 \\
\vdots & & \vdots \\
0 & \cdots & 0
\end{array}\right)
$$

one can break the gauge symmetry along $\gamma$ to $U(M) \times U(N-M)$, where the surviving $U(M)$ is the diagonal in $[U(M) \times U(M)] \times U(N-M) .{ }^{9}$ We conjecture that from the point of view of the gauge theory on $\mathcal{D}$, the only effect of the interaction with the background branes comes from the fact that

\footnotetext{
${ }^{8}$ The gauge theory has at least a $U(1)^{M}$ symmetry, and since the degeneracies are symmetric under the symmetric group $S_{M}$, we can organize them into characters of representations $\mathcal{Q}_{\mathrm{e}}^{\text {open }}$ of $U(M)$ (possibly with negative multiplicities).

${ }^{9}$ We are considering only the case $M<N$; ultimately we will be interested in taking $N$ large whereas $M$ stays finite.
} 
the $U(M)$ part of the gauge field along $\gamma$ is identified with the $U(M)$ gauge field on the background branes, via this Higgsing to the diagonal. We can account for this by inserting a $\delta$-function in the theory on $\mathcal{D}$, which freezes $M$ of the eigenvalues of the holonomy $\mathrm{e}^{i \oint_{\gamma} \mathcal{A}}$, identifying them with the holonomy on the background branes, which we call $\mathrm{e}^{i \phi}$. The Weyl invariant way to write this delta function is

$$
\delta_{M}\left(\mathrm{e}^{i \oint_{\gamma} \mathcal{A}}, \mathrm{e}^{i \phi}\right)=D\left(\oint_{\gamma} \mathcal{A}\right)^{-1} \sum_{\sigma \in S_{N}}(-)^{\sigma} \prod_{j=1}^{M} \delta\left(\left(\mathrm{e}^{i \oint_{\gamma} \mathcal{A}}\right)_{\sigma(j)}, \mathrm{e}^{i \phi_{j}}\right)
$$

where $D$ denotes the Vandermonde determinant (A.4).

Let us write $Z_{\mathrm{YM}}^{\mathrm{open}}\left(\varphi_{0}, \varphi_{2}, \phi\right)$ for the partition function with this operator inserted (here "open" refers to the fact that it is related to the open topological string). This partition function sums over the open D2-branes which end on the Lagrangian branes, as well as over the D0 and D2-brane charges which one had without the Lagrangian branes; so altogether we should have

$$
Z_{\mathrm{YM}}^{\text {open }}=Z_{\mathrm{BPS}}^{\text {open }}
$$

In this ensemble, the chemical potential $\varphi_{\mathrm{e}}^{\text {open }}$ for the open D2-branes should roughly be their mass. This mass is given by the area of the disc on which they are wrapped, which is related by supersymmetry to the Wilson line on the background branes; with this as motivation we write

$$
\varphi_{\mathrm{e}}^{\mathrm{open}}=2 \pi \phi / g_{s}
$$

To compute $Z_{\mathrm{YM}}^{\mathrm{open}}$ it is convenient to reduce from the twisted $\mathcal{N}=4$ theory on $\mathcal{D}$ to a $q$-deformed Yang-Mills theory on $\Sigma$, as was done in $[5,6]$. How does the operator insertion $\delta_{M}\left(\mathrm{e}^{i \oint_{\gamma} \mathcal{A}}, \mathrm{e}^{i \phi}\right)$ translate to the reduced theory? There are two cases to consider:

1. $\gamma$ lies in the fiber of $\mathcal{L}_{-p}$ over a point $P \in \Sigma$.

2. $\gamma$ lies on the Riemann surface $\Sigma$.

In either case, these Lagrangian branes can be locally modelled by the ones studied in refs. $[27,28]$. In case 1 , where $\gamma$ is in the fiber over $P$, the situation is basically straightforward: as explained in ref. [5], the flux $\oint_{\gamma} \mathcal{A}$ shows up in the $q$-deformed Yang-Mills theory on $\Sigma$ as a field $\Phi$. The operator we have to insert in the $q$-deformed theory is therefore

$$
\delta_{M}\left(\mathrm{e}^{i \Phi(P)}, \mathrm{e}^{i \phi}\right)
$$

The path integral gets localized on configurations where $\Phi$ is locally constant, so when there are no other operator insertions we can drop the $P$ and write $\delta_{M}\left(\mathrm{e}^{i \Phi}, \mathrm{e}^{i \phi}\right)$. 
In case 2 the situation is a bit trickier, because of a subtlety which also appeared in ref. [5]: namely, in performing the reduction one has to choose $p$ points $P_{i}$ on $\Sigma$, and at each such point one gets an operator corresponding to one unit of area in the Yang-Mills theory. The operator $\delta_{M}\left(\mathrm{e}^{i \oint_{\gamma} \mathcal{A}}, \mathrm{e}^{i \phi}\right)$ reduces to

$$
\delta_{M}\left(\mathrm{e}^{i \oint_{\gamma} A}, \mathrm{e}^{i \phi}\right)
$$

in two dimensions, but we have to specify how many of the $p$ points go on each side of $\gamma$. Therefore, there is a $\mathbb{Z}$-valued ambiguity in defining which operator we insert in the physical theory, parameterized by a choice of $p_{1}$ and $p_{2}$ with $p_{1}+p_{2}=p$. See figure 4 . This ambiguity should be understood as related to infrared regularization arising from the non-compactness of the situation; in the connection to the open topological string below, we will see that it is identified with the framing ambiguity.

\subsection{Specializing to genus zero}

Next we will investigate in detail the case when $\Sigma$ has genus zero. So we specialize to Type IIA on $X \times \mathbb{R}^{3,1}$, where

$$
X=\mathcal{O}(-p) \oplus \mathcal{O}(p-2) \rightarrow \mathbb{C P}^{1}
$$

with background D4-branes added on $L \times \mathbb{R}^{1,1}$. As we just explained, we can compute a mixed ensemble partition function $Z_{\mathrm{BPS}}^{\mathrm{open}}$ for this system by inserting an appropriate operator into the $q$-deformed Yang-Mills theory on an $S^{2}$ of area $p$. The parameters of the Yang-Mills theory are as in the

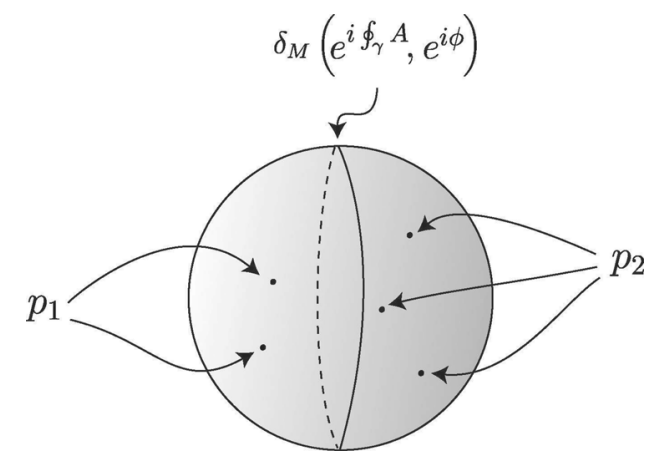

Figure 4: The operator $\delta_{M}\left(\mathrm{e}^{i \oint_{\gamma} A}, \mathrm{e}^{i \phi}\right)$ cuts $\Sigma$ into two pieces. 
closed case,

$$
\theta_{\mathrm{YM}}=\theta, \quad g_{\mathrm{YM}}^{2}=g_{s}, \quad q=\mathrm{e}^{-g_{s}} .
$$

We will show that for all $p_{1}, p_{2}$ we indeed have $Z_{\mathrm{YM}}^{\mathrm{open}}=\left|\psi_{\mathrm{top}}^{\mathrm{open}}\right|^{2}+\mathcal{O}\left(\mathrm{e}^{-N}\right)$. We will also show that the identification of $Z_{\mathrm{YM}}^{\text {open }}$ with $Z_{\mathrm{BPS}}^{\mathrm{open}}$ is consistent; namely, $Z_{\mathrm{BPS}}^{\mathrm{open}}$ should have an expansion where $\phi$ appears only in the form $\mathrm{e}^{-2 \pi \phi / g_{s}}$, and we will verify that $Z_{\mathrm{YM}}^{\mathrm{open}}$ indeed has such an expansion at least in the special case $p_{1}=p_{2}=1$. These two results together give evidence for our conjecture (5.6).

\subsection{Large $N$ factorization on $\mathcal{O}(-p) \oplus \mathcal{O}(p-2) \rightarrow \mathbb{C P}^{1}$}

We want to establish that

$$
Z_{\mathrm{YM}}^{\text {open }}=\left|\psi_{\text {top }}^{\text {open }}\right|^{2}+\mathcal{O}\left(\mathrm{e}^{-N}\right) .
$$

We compute $Z_{\mathrm{YM}}^{\mathrm{open}}$ using the gluing procedure described in Appendix B: namely, we construct the sphere by gluing two discs together with the operator $\delta_{M}\left(\mathrm{e}^{i \oint A}, \mathrm{e}^{i \phi}\right)$ in the middle. We use the fact that the Hilbert space of the two-dimensional Yang-Mills theory is factorized at large $N, \mathcal{H} \simeq \mathcal{H}_{+} \otimes \mathcal{H}_{-}$, and furthermore each component of the gluing procedure can be written in a factorized form. This factorization is described in detail in Appendix D; the computation of $Z_{\mathrm{YM}}^{\mathrm{open}}$ we give below basically consists of fetching various results from that appendix and putting them together. We then compare this with the known form of the topological string amplitude and find the desired factorization; the final result is given in (6.21).

\subsubsection{Branes in the base}

Let us first discuss case 2 , where to compute $Z_{\mathrm{YM}}^{\mathrm{open}}$ we have to insert a Wilson line freezing operator $\delta_{M}\left(\mathrm{e}^{i \oint A}, \mathrm{e}^{i \phi}\right)$. This operator cuts the sphere into two pieces, with discrete areas $p_{1}, p_{2}$ such that $p_{1}+p_{2}=p$. The gluing computation of $Z_{\mathrm{YM}}^{\mathrm{open}}$ involves a zero area disc, an annulus of area $p_{1}$, the operator $\delta_{M}\left(\mathrm{e}^{i \oint A}, \mathrm{e}^{i \phi}\right)$, an annulus of area $p_{2}$, and another zero area disc:

$$
Z_{\mathrm{YM}}^{\mathrm{open}}\left(N, g_{s}, \theta, \phi\right)=\left\langle\Psi_{0}\left|A_{p_{1}} \delta_{M}\left(\mathrm{e}^{i \oint A}, \mathrm{e}^{i \phi}\right) A_{p_{2}}\right| \Psi_{0}\right\rangle .
$$

Each of these pieces has been written in the factorized basis for $\mathcal{H}$ in Appendix D: the disc is given in (D.29), the annulus in (D.27), and the Wilson line freezing operator in (D.28). Plugging in these factorizations and doing a little rearranging, we obtain the factorized form of $Z_{\mathrm{YM}}^{\mathrm{open}}$, schematically 
$Z_{\mathrm{YM}}^{\mathrm{open}}=Z_{+} Z_{-}$, or more precisely (writing $q=\mathrm{e}^{-g_{s}}$ )

$$
\begin{aligned}
& Z_{\mathrm{YM}}^{\mathrm{open}}\left(N, g_{s}, \theta, \phi\right) \\
& \quad=Z_{\mathrm{YM}}^{0}\left(N, g_{s}, \theta, \phi\right) M(q)^{2} \eta(q)^{2 N} \times \sum_{l \in \mathbb{Z}, R_{1}^{\prime}, R_{2}^{\prime}}(-)^{\left|R_{1}^{\prime}\right|+\left|R_{2}^{\prime}\right|} \\
& \quad \times q^{\frac{1}{2}\left(p_{1}+p_{2}\right) l^{2}} \mathrm{e}^{i N l p \theta} Z_{+}^{R_{1}^{\prime}, R_{2}^{\prime}, l} Z_{-}^{R_{1}^{\prime}, R_{2}^{\prime}, l}+\mathcal{O}\left(\mathrm{e}^{-N}\right),
\end{aligned}
$$

where

$$
\begin{aligned}
& Z_{+}^{R_{1}^{\prime}, R_{2}^{\prime}, l}\left(N, g_{s}, \theta, \phi\right) \\
& =q^{\frac{1}{2} N\left(\left|R_{1}^{\prime}\right|+\left|R_{2}^{\prime}\right|\right)} \times \sum_{R_{1+}, R_{2+}, A_{+}} q^{\frac{1}{2} p_{1} \kappa_{R_{1+}}}+\frac{1}{2} p_{2} \kappa_{R_{2+}}+\left(\frac{1}{2} N\left(p_{1}-1\right)+l p_{1}\right) \\
& \quad \times\left|R_{1+}\right|+\left(\frac{1}{2} N\left(p_{2}-1\right)+l p_{2}\right)\left|R_{2+}\right| \times C_{0 R_{1}^{\prime} R_{1+}} C_{R_{2}^{\prime t} R_{2+} s_{R_{1+}} / A_{+}}\left(\mathrm{e}^{-i \phi}\right) \\
& \quad \times s_{R_{2+} / A_{+}}\left(\mathrm{e}^{i \phi}\right) \mathrm{e}^{i \theta\left(p_{1}\left|R_{1+}\right|+p_{2}\left|R_{2+}\right|\right)}
\end{aligned}
$$

and similarly

$$
\begin{aligned}
& Z_{-}^{R_{1}^{\prime}, R_{2}^{\prime}, l}\left(N, g_{s}, \theta, \phi\right) \\
& =q^{\frac{1}{2} N\left(\left|R_{1}^{\prime}\right|+\left|R_{2}^{\prime}\right|\right)} \sum_{R_{1-}, R_{2-}, A_{-}} \\
& \times q^{\frac{1}{2} p_{1} \kappa_{R_{1-}}+\frac{1}{2} p_{2} \kappa_{R_{2-}}+\left(\frac{1}{2} N\left(p_{1}-1\right)-l p_{1}\right)\left|R_{1-}\right|+\left(\frac{1}{2} N\left(p_{2}-1\right)-l p_{2}\right)\left|R_{2-}\right|} \\
& \times C_{0 R_{1}^{\prime t} R_{1-}} C_{R_{2}^{\prime} R_{2-} 0} s_{R_{1-} / A_{-}}\left(\mathrm{e}^{i \phi}\right) s_{R_{2-} / A_{-}}\left(\mathrm{e}^{-i \phi}\right) \mathrm{e}^{-i \theta\left(p_{1}\left|R_{1-}\right|+p_{2}\left|R_{2-}\right|\right)} .
\end{aligned}
$$

The normalization factor $Z_{\mathrm{YM}}^{0}\left(N, g_{s}, \theta, \phi\right)$ will be fixed below.

Now we want to interpret the chiral blocks $Z_{ \pm}^{R_{1}^{\prime}, R_{2}^{\prime}, l}(\phi)$ in terms of the topological string on $X$. This $X$ can be represented torically by the picture in figure 5 , in which we also indicate the Lagrangian cycle $L$ supporting $M$ branes, one supporting a stack of infinitely many ghost branes, and one with a stack of infinitely many ghost antibranes. (See e.g. [28] for a review of the meaning of toric pictures such as this one). The results of ref. [20] give 


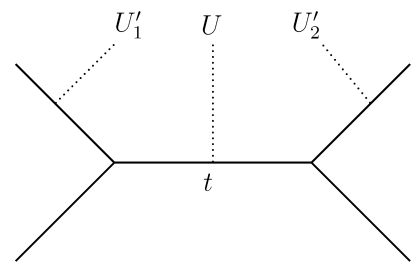

Figure 5: The vertex representation of $X=\mathcal{O}(-p) \oplus \mathcal{O}(p-2) \rightarrow \mathbb{C P}^{1}$, with a stack of $M$ branes with complexified holonomy $U=\mathrm{e}^{u}$, a stack of infinitely many ghost branes with complexified holonomy $U_{1}^{\prime}=\mathrm{e}^{u_{1}^{\prime}}$, and a stack of infinitely many ghost antibranes with complexified holonomy $U_{2}^{\prime}=\mathrm{e}^{u_{2}^{\prime}}$.

the topological string amplitude on this geometry as ${ }^{10} \quad$ (with $q=\mathrm{e}^{-g_{\mathrm{top}}}$ )

$$
\begin{aligned}
& \psi_{\text {top }}^{\mathrm{g}}\left(g_{\mathrm{top}}, t, u, u^{\prime}\right)=\psi_{\text {top }}^{0}\left(g_{\mathrm{top}}, t, u\right) \sum_{R_{1}, R_{2}, A, R_{1}^{\prime}, R_{2}^{\prime}}(-)^{\left|R_{2}^{\prime}\right|} s_{R_{1}^{\prime}}\left(\mathrm{e}^{u_{1}^{\prime}}\right) s_{R_{2}^{\prime}}\left(\mathrm{e}^{u_{2}^{\prime}}\right)
\end{aligned}
$$

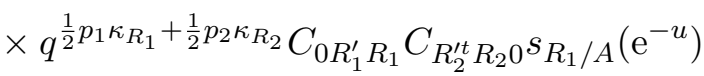

$$
\begin{aligned}
& \times s_{R_{2} / A}\left(\mathrm{e}^{u}\right)(-)^{p_{1}\left|R_{1}\right|+p_{2}\left|R_{2}\right|} \mathrm{e}^{-t\left|R_{2}\right|},
\end{aligned}
$$

with the choice of $p_{1}$ and $p_{2}$ (subject to the constraint $p_{1}+p_{2}=p$ ) related to the choice of framing on the Lagrangian branes. ${ }^{11}$ Similarly, if one swaps the ghost branes for ghost antibranes, one gets

$$
\begin{aligned}
& \psi_{\text {top }}^{\mathrm{a}}\left(g_{\text {top }}, t, u, u^{\prime}\right)=\psi_{\text {top }}^{0}\left(g_{\text {top }}, t, u\right) \sum_{R_{1}, R_{2}, A, R_{1}^{\prime}, R_{2}^{\prime}}(-)^{\left|R_{1}^{\prime}\right|} s_{R_{1}^{\prime}}\left(\mathrm{e}^{u_{1}^{\prime}}\right) s_{R_{2}^{\prime}}\left(\mathrm{e}^{u_{2}^{\prime}}\right)
\end{aligned}
$$

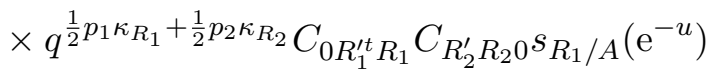

$$
\begin{aligned}
& \times s_{R_{2} / A}\left(\mathrm{e}^{u}\right)(-)^{p_{1}\left|R_{1}\right|+p_{2}\left|R_{2}\right|} \mathrm{e}^{-t\left|R_{2}\right|} .
\end{aligned}
$$

Now to relate the chiral blocks $Z_{ \pm}$which make up $Z_{\mathrm{YM}}$ to the topological string amplitudes, we define

$$
\begin{aligned}
t & =\frac{1}{2} N g_{s}(p-1)-i p \hat{\theta}, \\
u & =\frac{1}{2} N g_{s}\left(p_{1}-1\right)-i\left(p_{1} \hat{\theta}-\phi\right),
\end{aligned}
$$

\footnotetext{
${ }^{10}$ Using the result as it appears in ref. [20] one would actually get something slightly different from (6.14), namely, $R_{2} / A$ would be replaced by $R_{2}^{t} / A^{t}$, and there would be an extra overall factor $(-)^{\left|R_{2}\right|+|A|}$. This difference is due to a typo in ref. [20].

${ }^{11}$ Strictly speaking, ref. [20] considers the case $M=\infty$; but one can get finitely many branes by setting all but $M$ components of the $\mathrm{e}^{u}$ and $\mathrm{e}^{-u}$ appearing in (6.14) to zero.
} 


$$
\begin{aligned}
u_{1}^{\prime} & =\frac{1}{2} N g_{s}+i \phi_{1}^{\prime}, \\
u_{2}^{\prime} & =\frac{1}{2} N g_{s}+i \phi_{2}^{\prime}, \\
g_{\mathrm{top}} & =g_{s} .
\end{aligned}
$$

Here we introduced $\hat{\theta}=\theta+\pi$; this shift is meant to cancel the factor $(-)^{p|R|}$ in $(6.14) .{ }^{12}$

The desired factorization is then basically straightforward to check. One begins with (6.11) which expresses $Z_{\mathrm{YM}}$ in terms of the chiral blocks, then relates the chiral blocks to $\psi_{\text {top }}^{\mathrm{g}}$ and $\psi_{\text {top }}^{\mathrm{a}}$ with the above choice of parameters, and converts the sums over $R_{1}^{\prime}, R_{2}^{\prime}$ into integrals over $\phi_{1}^{\prime}, \phi_{2}^{\prime}$ as discussed in Section 3. This essentially gives

$$
\begin{aligned}
Z_{\mathrm{YM}}\left(N, g_{s}, \theta, \phi\right)= & \sum_{l \in \mathbb{Z}} \int d_{H} \phi_{1}^{\prime} d_{H} \phi_{2}^{\prime}\left(\psi_{\mathrm{top}}^{\mathrm{g}}\left(g_{s}, t+l p g_{s}, u+l p_{1} g_{s}, u^{\prime}\right)\right) \\
& \times \overline{\left(\psi_{\mathrm{top}}^{\mathrm{a}}\left(g_{s}, t-l p g_{s}, u-l p_{1} g_{s}, u^{\prime}\right)\right)} .
\end{aligned}
$$

In order to match the $l$-dependent terms in (6.11), though, one has to examine carefully the normalizations for the topological string and Yang-Mills amplitudes, as was done in $[5,6]$. For the topological string we write

$$
\psi_{\mathrm{top}}^{0}\left(g_{\mathrm{top}}, t\right)=M(q) \eta(q)^{2 t /(p-2) g_{\mathrm{top}}} \exp \left(-\frac{1}{6 p(p-2) g_{\mathrm{top}}^{2}} t^{3}+\frac{p-2}{24 p} t\right) \text {. }
$$

The meaning of this normalization factor was discussed in ref. [6]. For the Yang-Mills theory, we write

$$
Z_{\mathrm{YM}}^{0}\left(N, g_{s}, \theta, \phi\right)=\exp \left(\frac{g_{s}(p-2)^{2}}{24 p}\left(N-N^{3}\right)+N \frac{\hat{\theta}^{2} p}{2 g_{s}}\right) .
$$

Then the two chiral normalization factors multiply together to give the Yang-Mills normalization, up to some crucial $l$-dependent corrections:

$$
\begin{aligned}
& \psi_{\text {top }}^{0}\left(g_{s}, t+l p g_{s}, u+l p_{1} g_{s}\right) \overline{\psi_{\text {top }}^{0}\left(g_{s}, t-l p g_{s}, u-l p_{1} g_{s}\right)} \\
& =Z_{\mathrm{YM}}^{0}\left(N, g_{s}, \theta, \phi\right) M(q)^{2} \eta(q)^{2 N} q^{\frac{1}{2} p l^{2}} \mathrm{e}^{i N l p \theta} .
\end{aligned}
$$

These terms match the $l$-dependent terms in (6.11); they are exactly what is needed to make the factorization (6.21) work. So we have completed the

\footnotetext{
${ }^{12}$ The apparent asymmetry between $p_{1}$ and $p_{2}$ comes from the fact that we chose $u$ to represent the complexified area of the disc which ends on the Lagrangian branes from the left; the disc which ends on them from the right has area $t-u=\frac{1}{2} N g_{s}\left(p_{2}-1\right)-$ $i\left(p_{2} \hat{\theta}+\phi\right)$.
} 
factorization in case 2, corresponding to D-branes which intersect the base $\mathbb{C P}^{1}$ in $X$.

\subsubsection{Branes in the fiber}

We can also consider case 1, corresponding to D-branes which meet the fiber of $\mathcal{O}(-p) \rightarrow \mathbb{C P}^{1}$. In this case, in the Yang-Mills theory we insert the dual Wilson line freezing operator $\delta_{M}\left(\mathrm{e}^{i \Phi}, \mathrm{e}^{i \phi}\right)$ at a point of $\mathbb{C P}^{1}$. Our discussion here will be more brief since the proof of the factorization runs along the same lines as case 2 above.

Again, we compute the Yang-Mills amplitude by gluing: we have to glue a disc containing the operator $\delta_{M}\left(\mathrm{e}^{i \Phi}, \mathrm{e}^{i \phi}\right)$, an annulus of area $p$, and another disc, obtaining

$$
Z_{\mathrm{YM}}^{\mathrm{open}}\left(N, g_{s}, \theta, \phi\right)=\left\langle\Psi_{\phi}\left|A_{p}\right| \Psi_{0}\right\rangle .
$$

Using the factorization results (D.27), (D.29) and (D.30) this becomes

$$
\begin{aligned}
& Z_{\mathrm{YM}}^{\text {open }}\left(N, g_{s}, \theta, \phi\right) \\
& =Z_{\mathrm{YM}}^{0}\left(N, g_{s}, \theta, \phi\right) M(q)^{2} \eta(q)^{2 N} \sum_{l, m \in \mathbb{Z}, R_{1}^{\prime}, R_{2}^{\prime}}(-)^{\left|R_{1}\right|+\left|R_{2}\right|} q^{\frac{1}{2} p l^{2}} \mathrm{e}^{i N l p \theta} \\
& \quad \times \operatorname{det}\left(\mathrm{e}^{i m \phi}\right) Z_{+}^{R_{1}^{\prime}, R_{2}^{\prime}, l, m} Z_{-}^{R_{1}^{\prime}, R_{2}^{\prime}, l, m}+\mathcal{O}\left(\mathrm{e}^{-N}\right),
\end{aligned}
$$

with

$$
\begin{aligned}
& Z_{+}^{R_{1}^{\prime}, R_{2}^{\prime}, l, m}\left(N, g_{s}, \theta, \phi\right) \\
& =q^{\frac{1}{2} N\left(\left|R_{1}^{\prime}\right|+\left|R_{2}^{\prime}\right|\right)} \sum_{R_{+}, T_{+}} q^{\frac{1}{2} p \kappa_{R_{+}}+\frac{1}{2} \kappa_{T_{+}}+\left(\frac{1}{2} N(p-2)+l p-m\right)\left|R_{+}\right|+\left(-\frac{1}{2} N-l\right)\left|T_{+}\right|} \\
& \quad \times C_{T_{+} R_{1}^{\prime} R_{+}} C_{R_{2}^{\prime t} R_{+} 0} s_{T_{+}^{t}}\left(\mathrm{e}^{-i \phi}\right) \mathrm{e}^{i \theta p\left|R_{+}\right|},
\end{aligned}
$$

and similarly

$$
\begin{aligned}
& Z_{-}^{R_{1}^{\prime}, R_{2}^{\prime}, l, m}\left(N, g_{s}, \theta, \phi\right) \\
& =q^{\frac{1}{2} N\left(\left|R_{1}^{\prime}\right|+\left|R_{2}^{\prime}\right|\right)} \sum_{R_{-}, T_{-}} q^{\frac{1}{2} p \kappa_{R_{-}}+\frac{1}{2} \kappa_{T_{-}}+\left(\frac{1}{2} N(p-2)-l p+m\right)\left|R_{-}\right|+\left(-\frac{1}{2} N+l\right)\left|T_{-}\right|} \\
& \quad \times C_{T_{-} R_{1}^{\prime t} R_{-}} C_{R_{2}^{\prime} R_{-} 0} s_{T_{-}^{t}}\left(\mathrm{e}^{i \phi}\right) \mathrm{e}^{-i \theta p\left|R_{-}\right|} .
\end{aligned}
$$

As in case 2, we can now interpret these chiral blocks in terms of the topological string on $X$ with $M$ Lagrangian branes, now inserted on the external leg as indicated in figure 6. Again from ref. [20], the topological partition function in this geometry (with a particular choice of framing on the $M$ 


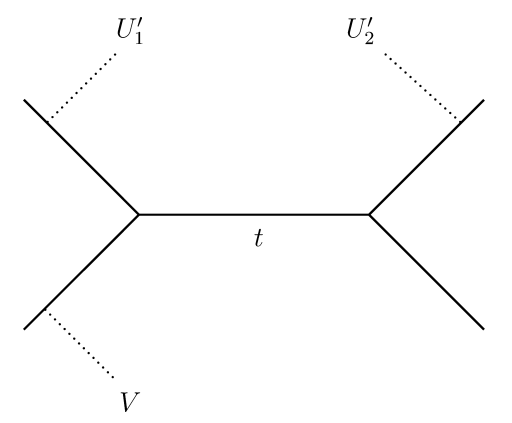

Figure 6: The vertex representation of $X$, with a stack of $M$ Lagrangian branes with complexified holonomy $V=\mathrm{e}^{v}$, a stack of infinitely many ghost branes with complexified holonomy $U_{1}^{\prime}=\mathrm{e}^{u_{1}^{\prime}}$, and a stack of infinitely many ghost antibranes with complexified holonomy $U_{2}^{\prime}=\mathrm{e}^{u_{2}^{\prime}}$.

external branes) is

$$
\begin{aligned}
\psi_{\text {top }}^{\mathrm{g}}\left(g_{\text {top }}, t, v, u^{\prime}\right)= & \psi_{\text {top }}^{0}\left(g_{\text {top }}, t, v\right) \sum_{R, T} C_{T R_{1}^{\prime} R} C_{R_{2}^{\prime} R 0} s_{R_{1}^{\prime}}\left(\mathrm{e}^{u_{1}^{\prime}}\right) s_{R_{2}^{\prime}}\left(\mathrm{e}^{u_{2}^{\prime}}\right) \\
& \times(-)^{p|R|} q^{\frac{1}{2} p \kappa_{R}+\frac{1}{2} \kappa_{T}} \mathrm{e}^{-t|R|} s_{T^{t}}\left(\mathrm{e}^{-v}\right),
\end{aligned}
$$

and similarly one can compute $\psi_{\text {top }}^{\mathrm{a}}$ with the ghost branes exchanged for antibranes. Now define

$$
\begin{aligned}
t & =\frac{1}{2} N g_{s}(p-2)-i \theta p, \\
u_{1}^{\prime} & =\frac{1}{2} N g_{s}+i \phi_{1}^{\prime}, \\
u_{2}^{\prime} & =\frac{1}{2} N g_{s}+i \phi_{2}^{\prime}, \\
v & =-\frac{1}{2} N g_{s}+i \phi, \\
g_{\text {top }} & =g_{s} .
\end{aligned}
$$

With this substitution and a suitable normalization, one can relate (6.29) to the chiral blocks appearing in the factorization (6.26), similarly to what was done above in case 2 , obtaining

$$
\begin{aligned}
& Z_{\mathrm{YM}}\left(N, g_{s}, \theta, \phi\right) \\
& =\sum_{l, m \in \mathbb{Z}} \int d_{H} \phi_{1}^{\prime} d_{H} \phi_{2}^{\prime}\left(\psi_{\text {top }}^{\mathrm{g}}\left(g_{s}, t+l p g_{s}, v-l g_{s}, u_{1}^{\prime}-m g_{s}, u_{2}^{\prime}\right)\right) \\
& \quad \times \overline{\left(\psi_{\text {top }}^{\mathrm{a}}\left(g_{s}, t-l p g_{s}, v+l g_{s}, u_{1}^{\prime}+m g_{s}, u_{2}^{\prime}\right)\right)} .
\end{aligned}
$$

So finally we have found that $Z_{\mathrm{YM}}^{\text {open }}=\left|\psi_{\text {top }}^{\text {open }}\right|^{2}+\mathcal{O}\left(\mathrm{e}^{-N}\right)$, both for branes in the fiber and in the base. 


\subsection{Summing open D2-branes on $\mathcal{O}(-1) \oplus \mathcal{O}(-1) \rightarrow \mathbb{C P}^{1}$}

Next we want to verify that $Z_{\mathrm{YM}}^{\text {open }}$ can indeed be interpreted as counting open D2-branes with the chemical potential $\varphi_{\mathrm{e}}^{\mathrm{open}}=2 \pi \phi / g_{s}$. We consider case 2 , where we have a Wilson line freezing operator $\delta_{M}\left(\mathrm{e}^{i \oint_{\gamma} A}, \mathrm{e}^{i \phi}\right)$ cutting the sphere into two pieces, with discrete areas $p_{1}, p_{2}$ such that $p_{1}+p_{2}=p$, and further specialize to the case $p_{1}=p_{2}=1$.

As we did in the previous section, we compute the partition function $Z_{\mathrm{YM}}^{\text {open }}$ of this Yang-Mills theory using the gluing procedure described in Appendix B: namely, we construct the sphere by gluing two discs together with the operator $\delta_{M}\left(\mathrm{e}^{i \oint A}, \mathrm{e}^{i \phi}\right)$ in the middle. However, unlike above where we used the splitting $\mathcal{H}=\mathcal{H}_{+} \otimes \mathcal{H}_{-}$to see the large $N$ factorization of $Z_{\mathrm{YM}}^{\text {open }}$, in this section we will write the explicit formula for $Z_{\mathrm{YM}}^{\mathrm{open}}$ at finite $N$.

The wave function of the $q$-deformed two-dimensional Yang-Mills theory on the disc is a function of the eigenvalues $\xi$ of the Wilson line around the boundary, evaluated in Appendix C:

$$
\Psi(\xi)=\mathrm{e}^{-N g_{s} / 24} \Theta_{N}\left(\frac{1}{2 \pi}(\xi+\theta), \frac{i g_{s}}{2 \pi}\right)
$$

where $\Theta_{N}$ denotes the theta function of $\mathbb{Z}^{N}$,

$$
\Theta_{N}(z, \tau)=\sum_{\gamma \in \mathbb{Z}^{N}} \mathrm{e}^{\pi i \tau\|\gamma\|^{2}} \mathrm{e}^{2 \pi i\langle\gamma, z\rangle} \quad \text { for } z \in \mathbb{R}^{N}, \operatorname{Im} \tau>0
$$

In our case, we want to glue two such disc wave functions $\Psi_{1}(\xi), \Psi_{2}(\xi)$ to one another with $\delta_{M}\left(\mathrm{e}^{i \oint A}, \mathrm{e}^{i \phi}\right)$ sandwiched in the middle. The result of this gluing is given in (B.8), but we need a little notation first: we divide the lattice $\mathbb{Z}^{N}$ into $\mathbb{Z}^{N-M} \oplus \mathbb{Z}^{M}$, and correspondingly divide $\xi$ into $\zeta$ and $\phi$, with $N-M$ and $M$ components, respectively. Then the result of the gluing is

$$
Z_{\mathrm{YM}}^{\mathrm{open}}\left(N, g_{s}, \theta, \phi\right)=\int_{[0,2 \pi]^{N-M}} \frac{d \zeta}{2 \pi}|D(\zeta)|^{2} \Psi_{1}(-\zeta,-\phi) \Psi_{2}(\zeta, \phi)
$$

Because of the simple form of the wave function, the $\zeta$ dependence and $\phi$ dependence decouple, namely

$$
\Psi(\zeta, \phi)=\mathrm{e}^{-N g_{s} / 24} \Theta_{N-M}\left(\frac{1}{2 \pi}(\zeta+\theta), \frac{i g_{s}}{2 \pi}\right) \Theta_{M}\left(\frac{1}{2 \pi}(\phi+\theta), \frac{i g_{s}}{2 \pi}\right)
$$


So write

$$
\begin{aligned}
f_{N-M}\left(\theta, g_{s}\right)= & \int \frac{d \zeta}{2 \pi}|D(\zeta)|^{2} \Theta_{N-M}\left(\frac{1}{2 \pi}(\zeta+\theta), \frac{i g_{s}}{2 \pi}\right) \\
& \times \Theta_{N-M}\left(\frac{1}{2 \pi}(-\zeta+\theta), \frac{i g_{s}}{2 \pi}\right) .
\end{aligned}
$$

Then (6.38) becomes

$$
\begin{aligned}
Z_{\mathrm{YM}}^{\mathrm{open}}\left(N, g_{s}, \theta, \phi\right)= & \mathrm{e}^{-N g_{s} / 12} f_{N-M}\left(\theta, g_{s}\right) \Theta_{M}\left(\frac{1}{2 \pi}(\phi+\theta), \frac{i g_{s}}{2 \pi}\right) \\
& \times \Theta_{M}\left(\frac{1}{2 \pi}(-\phi+\theta), \frac{i g_{s}}{2 \pi}\right) .
\end{aligned}
$$

Now we can use the Poisson resummation property of the theta function,

$$
\Theta_{M}(z, \tau)=\left(\frac{i}{\tau}\right)^{M / 2} \mathrm{e}^{-\pi i\|z\|^{2} / \tau} \Theta_{M}(z / \tau,-1 / \tau),
$$

to obtain

$$
\begin{aligned}
Z_{\mathrm{YM}}^{\mathrm{open}}\left(N, g_{s}, \theta, \phi\right)= & \mathrm{e}^{-N g_{s} / 12} f_{N-M}\left(\theta, g_{s}\right)\left(\frac{2 \pi}{g_{s}}\right)^{M} \mathrm{e}^{-\frac{1}{2 g_{s}}\left(\|\phi+\theta\|^{2}+\|\phi-\theta\|^{2}\right)} \\
& \times \Theta_{M}\left(-\frac{i}{g_{s}}(\phi+\theta), \frac{2 \pi i}{g_{s}}\right) \Theta_{M}\left(-\frac{i}{g_{s}}(-\phi+\theta), \frac{2 \pi i}{g_{s}}\right) .
\end{aligned}
$$

Expanding out these theta functions then gives $Z_{\mathrm{YM}}^{\text {open }}\left(N, g_{s}, \theta, \phi\right)$ as an expansion in $\mathrm{e}^{-2 \pi \phi / g_{s}}$, up to a prefactor $\mathrm{e}^{-\frac{1}{g_{s}}\|\phi\|^{2}}$. So up to this prefactor, we have verified that $Z_{\mathrm{YM}}^{\mathrm{open}}$ can indeed be interpreted as counting open D2-branes with the chemical potential $\varphi_{2}^{\text {open }}=2 \pi \phi / g_{s}$.

For completeness, let us briefly consider the leftover factor $f_{N-M}\left(\theta, g_{s}\right)$. Writing out using (A.4)

$$
|D(\zeta)|^{2}=\sum_{\sigma, \sigma^{\prime} \in S_{N-M}}(-)^{\sigma \sigma^{\prime}} \mathrm{e}^{i\left\langle\zeta, \sigma(\rho)-\sigma^{\prime}(\rho)\right\rangle}
$$

(where $\rho=\rho_{N-M}$ ) and evaluating the integral using the definitions of the theta functions gives

$$
\begin{aligned}
f_{N-M}\left(\theta, g_{s}\right)= & \sum_{\sigma, \sigma^{\prime} \in S_{N-M}}(-)^{\sigma \sigma^{\prime}} \mathrm{e}^{-\frac{1}{2} g_{s}\left\|\sigma(\rho)-\sigma^{\prime}(\rho)\right\|^{2}} \\
& \times \Theta_{N-M}\left(\frac{1}{2 \pi}\left(-2 \theta+i g_{s}\left(\sigma(\rho)-\sigma^{\prime}(\rho)\right)\right), \frac{i g_{s}}{\pi}\right) .
\end{aligned}
$$

So this can also be resummed to give an expansion in $\mathrm{e}^{-4 \pi^{2} / g_{s}}$ and $\mathrm{e}^{-2 \pi \theta / g_{s}}$, as one expects from the closed string sector of the conjecture. 


\section{Acknowledgments}

We would like to thank Jacques Distler, Noam Elkies, Sergei Gukov, Marcos Mariño, Shiraz Minwalla, Luboš Motl, Hirosi Ooguri and Natalia Saulina for useful discussions. The research of M.A. was supported in part by a DOE OJI Award and an Alfred P. Sloan Foundation fellowship. The research of A.N. and C.V. was supported in part by NSF grants PHY-0244821 and DMS-0244464.

\section{Appendix A Group theory}

In this appendix, we summarize our group theory conventions and a few useful formulas.

We use script letters $\mathcal{R}, \mathcal{P}, \mathcal{Q}, \ldots$ to denote representations of unitary groups such as $U(N)$, and capital letters $R, P, Q, \ldots$ to denote Young diagrams. Often Young diagrams will appear as the chiral and anti-chiral parts $R_{ \pm}$of a representation $\mathcal{R}=R_{+} \overline{R_{-}}[l]$, as described in Appendix D.

The weight lattice of $U(N)$ is $\mathbb{Z}^{N}$, with its standard inner product $\langle$,$\rangle . A$ highest weight representation $\mathcal{R}$ is characterized by a weight $\left(r_{1}, \ldots, r_{N}\right) \in$ $\mathbb{Z}^{N}$, in a particular Weyl chamber; we make the standard choice of Weyl chamber, given by the constraint $r_{1} \geq \cdots \geq r_{N}$. With this choice, the entries $r_{i}$ correspond to the lengths of the rows of the extended Young diagram for the representation $\mathcal{R}$. The Weyl group $W$ of $U(N)$ is the symmetric group, $W \simeq S_{N}$, which permutes the entries of $\mathbb{Z}^{N}$ in the obvious way.

We will use the symbol $\mathcal{R}$ both for the representation and for its highest weight. It is also convenient to introduce the symbol $\hat{\mathcal{R}}$ for $\mathcal{R}+\rho$, where $\rho$ is half the sum of the positive roots of $U(N)$, concretely

$$
\rho=\frac{1}{2}(N-1, N-3, \ldots, 3-N, 1-N) .
$$

We also write $\mathbf{1}$ for the "unit" vector,

$$
\mathbf{1}=(1,1, \ldots, 1,1) \text {. }
$$

With this notation we can write the Weyl character formula ${ }^{13}$

$$
\operatorname{Tr}_{\mathcal{R}}\left(\mathrm{e}^{i \xi}\right)=D(\xi)^{-1} \sum_{\sigma \in S_{N}}(-)^{\sigma} \mathrm{e}^{i\langle\hat{\mathcal{R}}, \sigma(\xi)\rangle},
$$

\footnotetext{
${ }^{13}$ When $N$ is odd, $D(\xi)^{-1}$ and $\sum_{\sigma \in S_{N}}(-)^{\sigma} \mathrm{e}^{i\langle\hat{\mathcal{R}}, \sigma(\xi)\rangle}$ are not quite well defined as functions of the eigenvalues $\mathrm{e}^{i \xi}$ - they change sign under $\xi_{i} \rightarrow \xi_{i}+2 \pi$. Nevertheless their product is still well defined.
} 
where the denominator $D(\xi)$ is

$$
D(\xi)=\sum_{\sigma \in S_{N}}(-)^{\sigma} \mathrm{e}^{i\langle\xi, \sigma(\rho)\rangle}=\prod_{i<j}\left(\mathrm{e}^{i\left(\xi_{i}-\xi_{j}\right) / 2}-\mathrm{e}^{-i\left(\xi_{i}-\xi_{j}\right) / 2}\right) .
$$

In computing the $q$-deformed Yang-Mills amplitudes, we will need to use the Hopf link invariant $S_{\mathcal{P} \mathcal{Q}}$ of the level $k$ Chern-Simons theory with gauge group $U(N)$. Define $g_{s}=\frac{2 \pi}{N+k}$. There is a formula expressing $S_{\mathcal{P} \mathcal{Q}}$ as a sum over the Weyl group $W \simeq S_{N}$ :

$$
S_{\mathcal{P} \mathcal{Q}}=\mathrm{e}^{-g_{s}\left(\|\rho\|^{2}+N / 24\right)} \sum_{\sigma \in S_{N}}(-)^{\sigma} \mathrm{e}^{g_{s}\langle\hat{\mathcal{P}}, \sigma(\hat{\mathcal{Q}})\rangle} .
$$

(The standard formulas for $S_{\mathcal{P} \mathcal{Q}}$ include a different normalization, but in the context where we will use $S_{\mathcal{P} \mathcal{Q}}$ we will absorb this in other normalization factors).

For any $N_{1}, N_{2}$ with $N_{1}+N_{2}=N$, let $\mathcal{Q}$ label a representation of $U\left(N_{1}\right)$ and $\mathcal{A}$ a representation of $U\left(N_{2}\right)$, whereas $\mathcal{R}$ is a representation of $U(N)$; then we define the branching coefficients $\mathcal{B}_{\mathcal{Q} \mathcal{A}}^{\mathcal{R}}$ by the rule that $\mathcal{R}$ decomposes under $U\left(N_{1}\right) \times U\left(N_{2}\right)$ as

$$
\mathcal{R} \rightarrow \bigoplus_{\mathcal{Q}, \mathcal{A}} \mathcal{B}_{\mathcal{Q} \mathcal{A}}^{\mathcal{R}}[\mathcal{Q}, \mathcal{A}]
$$

We fix the normalization of the Casimir operators of $U(N)$ as follows: in a representation $\mathcal{R}$ with highest weight $\left(r_{1}, \ldots, r_{N}\right)$ :

$$
\begin{aligned}
& C_{1}(\mathcal{R})=\langle\hat{\mathcal{R}}, \mathbf{1}\rangle=\sum_{i} r_{i}, \\
& C_{2}(\mathcal{R})=\|\hat{\mathcal{R}}\|^{2}-\|\rho\|^{2}=\sum_{i} r_{i}\left(r_{i}+N+1-2 i\right) .
\end{aligned}
$$

We write $N_{R_{1} R_{2}}^{R}$ for the usual Littlewood-Richardson numbers, and also use a slight generalization which we write $N_{R_{1} \cdots R_{k}}^{R}$. These numbers can be defined in various equivalent ways - for example, if we think of the Young diagrams $R_{i}$ and $R$ as representations of $G L(\infty)$, they are the tensor product coefficients, i.e.

$$
R_{1} \otimes \cdots \otimes R_{k}=\bigoplus_{R} N_{R_{1} \cdots R_{k}}^{R} R
$$

In particular, $N_{R_{1} \cdots R_{k}}^{R}=0$ unless $\sum_{i=1}^{k}\left|R_{i}\right|=|R|$, where $|R|$ denotes the total number of boxes in the diagram $R$.

We write $s_{R}(x)$ for the "Schur function" associated with the Young diagram $R$ : this is a symmetric polynomial in infinitely many variables, $x=$ $\left(x_{1}, x_{2}, \ldots\right)$. It can be defined in various equivalent ways; one convenient way to think of it is as the character of the $\operatorname{Mat}(\infty, \mathbb{C})$ representation associated 
to $R$, evaluated on the diagonal matrix with entries $\left(x_{1}, x_{2}, \ldots\right)$. There is a bilinear inner product $\langle$,$\rangle on the ring of symmetric polynomials for which$ the Schur functions form an orthonormal basis, $\left\langle s_{R}, s_{S}\right\rangle=\delta_{R S}$; in terms of this inner product $N_{R_{1} \cdots R_{k}}^{R}=\left\langle\prod_{i=1}^{k} s_{R_{i}}, s_{R}\right\rangle$. Viewing the $x_{i}$ as eigenvalues, the inner product can be written as a formal integral of class functions over $U(\infty)$ (interpreted as an inverse limit of finite-dimensional groups with their normalized Haar measures),

$$
\langle f, g\rangle=\int d_{\mathrm{H}} \xi f\left(\mathrm{e}^{-i \xi}\right) g\left(\mathrm{e}^{i \xi}\right) .
$$

We also use the "skew Schur functions" $s_{R / A}(x)$, defined by

$$
s_{R / A}(x)=\sum_{Q} N_{Q A}^{R} s_{Q}(x)
$$

See ref. [29] for much more on Schur functions and skew Schur functions.

We also introduce an analog of the skew Schur function, a "skew trace" involving the branching $U(N) \rightarrow U\left(N_{1}\right) \times U\left(N_{2}\right)$ where $N_{1}+N_{2}=N$ : this is a rule by which a representation of $U(N)$ and a representation of $U\left(N_{2}\right)$ induce a class function on $U\left(N_{1}\right)$, which we define by

$$
\operatorname{Tr}_{\mathcal{R} / \mathcal{A}}(U)=\sum_{\mathcal{Q}} \mathcal{B}_{\mathcal{Q} \mathcal{A}}^{\mathcal{R}} \operatorname{Tr}_{\mathcal{Q}}(U)
$$

Here $\mathcal{R}, \mathcal{Q}, \mathcal{A}$ denote the representations of $U(N), U\left(N_{1}\right), U\left(N_{2}\right)$, respectively; $\mathcal{B}$ denotes the branching coefficients defined in (A.6); and $U \in U\left(N_{1}\right)$.

We will frequently encounter sums $\sum_{R^{\prime}}$ over the set of all Young diagrams. A particularly useful identity for reducing such sums is

$$
\sum_{R^{\prime}, S^{\prime}}(-)^{\left|R^{\prime}\right|} N_{R^{\prime} S^{\prime} A_{1} \cdots A_{a}}^{A} N_{R^{\prime t} S^{\prime} B_{1} \cdots B_{b}}^{B}=N_{A_{1} \cdots A_{a}}^{A} N_{B_{1} \cdots B_{b}}^{B}
$$

One can prove (A.13) using the "Cauchy identities" for Schur functions, given e.g. in ref. [29],

$$
\begin{aligned}
\sum_{S^{\prime}} s_{S^{\prime}}(x) s_{S^{\prime}}(y) & =\prod_{i=1}^{\infty} \prod_{j=1}^{\infty}\left(1-x_{i} y_{j}\right)^{-1}, \\
\sum_{R^{\prime}}(-)^{\left|R^{\prime}\right|} s_{R^{\prime}}(x) s_{R^{\prime t}}(y) & =\prod_{i=1}^{\infty} \prod_{j=1}^{\infty}\left(1-x_{i} y_{j}\right) .
\end{aligned}
$$




\section{Appendix B The $q$-deformed 2-d Yang-Mills theory}

In this section, we review some facts about two-dimensional Yang-Mills theory and its $q$-deformed cousin. We begin with the two-dimensional Euclidean Yang-Mills action ${ }^{14}$ for gauge group $G=U(N)$,

$$
S_{\mathrm{YM}}=\frac{1}{2 g_{\mathrm{YM}}^{2}}\left(\int_{\Sigma} d^{2} x \operatorname{Tr} F \wedge * F+\theta_{\mathrm{YM}} \int_{\Sigma} \operatorname{Tr} F\right)
$$

It is often convenient to rewrite (B.1) by introducing an additional adjointvalued scalar field $\Phi$, which enters the action quadratically: namely, (B.1) is equivalent to

$$
S_{\mathrm{YM}}=\frac{1}{2 g_{\mathrm{YM}}^{2}}\left(2 \int_{\Sigma} \operatorname{Tr} \Phi F-\int_{\Sigma} \mu \operatorname{Tr} \Phi^{2}+\theta_{\mathrm{YM}} \int_{\Sigma} \mu \operatorname{Tr} \Phi\right)
$$

where $\mu$ is the area element on $\Sigma$. Once we have introduced this $\Phi$ we can define the $q$-deformed theory: we use the same action $S_{\mathrm{YM}}$, but we consider the fundamental variables to be the gauge connection and $\mathrm{e}^{i \Phi}$, rather than the gauge connection and $\Phi$. More precisely, since there is an ambiguity in recovering $\Phi$ from $\mathrm{e}^{i \Phi}, S_{\mathrm{YM}}$ is not well defined as a function of $\Phi$; to get a well-defined expression inside the path integral one has to sum $\mathrm{e}^{-S_{\text {YM }}}$ over all "images" $\Phi$. Equivalently, we integrate over all $\Phi$, not just a fundamental domain, but we use the measure appropriate for an integral over $\mathrm{e}^{i \Phi}$. This construction gives the $q$-deformed theory with $q=\mathrm{e}^{-g_{\mathrm{YM}}^{2}}$, which is the one that naturally occurs in this article; to get a different value of $q$ one would change the periodicity of $\Phi$.

The partition function can be computed in various ways; here we will focus on the computation by cutting and pasting. In the case of the undeformed Yang-Mills theory, this procedure was reviewed in ref. [30]; our treatment will be briefer, and is intended mostly to recall the new features that appear in the $q$-deformed case, as described in ref. [6].

To get the cutting-and-pasting procedure started one first needs to know the Hilbert space $\mathcal{H}$ of the theory on $S^{1}$; as for the usual 2-d Yang-Mills theory, it is simply the space of class functions $\Psi(g)$, with $g \in G$ interpreted as the holonomy of the connection around $S^{1}$. The path integral over a surface with boundary $S^{1}$ thus gives a state $\Psi(g)$. Two such surfaces can

\footnotetext{
${ }^{14}$ Note that our convention for $\theta_{\mathrm{YM}}$ is not the usual one; $\theta_{\mathrm{YM}}^{\mathrm{usual}}=\frac{i \pi}{g_{\mathrm{YM}}^{2}} \theta_{\mathrm{YM}}$.
} 
be glued using the rule ${ }^{15}$

$$
\left\langle\Psi_{1} \mid \Psi_{2}\right\rangle=\int_{G} d_{\mathrm{H}} g \Psi_{1}\left(g^{-1}\right) \Psi_{2}(g)
$$

with $d_{\mathrm{H}} g$ the Haar measure. When $G=U(N)$ we can write these wave functions more concretely as functions of the eigenvalues $e^{i \xi_{i}}$, totally symmetric under the permutation group $S_{N}$, and the gluing rule becomes

$$
\left\langle\Psi_{1} \mid \Psi_{2}\right\rangle=\int_{[0,2 \pi]^{N}} \frac{d \xi}{2 \pi}|D(\xi)|^{2} \Psi_{1}(-\xi) \Psi_{2}(\xi),
$$

where as in (A.4)

$$
D(\xi)=\prod_{i<j}\left(\mathrm{e}^{i\left(\xi_{i}-\xi_{j}\right) / 2}-\mathrm{e}^{-i\left(\xi_{i}-\xi_{j}\right) / 2}\right) .
$$

A convenient basis of $\mathcal{H}$ (which in particular diagonalizes the Hamiltonian) is given by the characters $\operatorname{Tr}_{\mathcal{R}}(g)$ as $\mathcal{R}$ runs over all representations of $G$. In that basis the gluing rule becomes

$$
\left\langle\mathcal{R}_{1} \mid \mathcal{R}_{2}\right\rangle=\delta_{\mathcal{R}_{1} \mathcal{R}_{2}}
$$

Next we need the partition function on a few elementary surfaces, from which any $\Sigma$ of interest to us can be pasted together.

The annulus. The annulus of area $a$ has two boundaries, so it gives an operator $A_{a}: \mathcal{H} \rightarrow \mathcal{H}$. The gluing rule for an annulus can be obtained directly from the action by working out the Hamiltonian; it is [6]

$$
\left\langle\mathcal{R}_{1}\left|A_{a}\right| \mathcal{R}_{2}\right\rangle=\delta_{\mathcal{R}_{1} \mathcal{R}_{2}} \mathrm{e}^{-a\left(\frac{1}{2} g_{\mathrm{YM}}^{2} C_{2}(\mathcal{R})-i \theta_{\mathrm{YM}} C_{1}(\mathcal{R})\right)} .
$$

The Wilson line freezing operator. As discussed in Section 5, we will be particularly interested in computing amplitudes involving a particular operator, written $\delta_{M}\left(\mathrm{e}^{i \oint A}, \mathrm{e}^{i \phi}\right)$, which has the effect of freezing $M$ of the eigenvalues along a Wilson loop to the values $\mathrm{e}^{i \phi_{1}}, \ldots, \mathrm{e}^{i \phi_{M}}$. A natural guess for the gluing rule with $\delta_{M}\left(\mathrm{e}^{i \oint A}, \mathrm{e}^{i \phi}\right)$ inserted can be obtained by splitting up the $N$ eigenvalues $\xi_{i}$ into $(\underbrace{\zeta}_{N-M}, \underbrace{\phi}_{M})$ : namely, one freezes the $\phi$ eigenvalues in

\footnotetext{
${ }^{15}$ We will always use the notation $\langle\mid\rangle$ to stand for the (linear) gluing rule rather than the (sesquilinear) inner product on the Hilbert space. The two are the same when acting on real linear combinations of the characters $\operatorname{Tr}_{\mathcal{R}}(g)$ but differ for complex linear combinations.
} 
the gluing rule (B.4) and integrates only over the $\zeta$ eigenvalues, obtaining

$$
\left\langle\Psi_{1}\left|\delta_{M}\left(\mathrm{e}^{i \oint A}, \mathrm{e}^{i \phi}\right)\right| \Psi_{2}\right\rangle=\int_{[0,2 \pi]^{N-M}} \frac{d \zeta}{2 \pi}|D(\zeta)|^{2} \Psi_{1}(-\zeta,-\phi) \Psi_{2}(\zeta, \phi) .
$$

This integral has an interpretation in the representation basis. Namely, suppose $\Psi_{j}(\xi)=\operatorname{Tr}_{\mathcal{R}_{j}}\left(\mathrm{e}^{i \xi}\right)$. Then decomposing $\mathcal{R}_{j}$ under $U(N-M) \times U(M)$ gives

$$
\Psi_{j}(\xi)=\sum_{\mathcal{A}_{j}, \mathcal{Q}_{j}} \mathcal{B}_{\mathcal{A}_{j} \mathcal{Q}_{j}}^{\mathcal{R}_{j}} \operatorname{Tr}_{\mathcal{A}_{j}}\left(\mathrm{e}^{i \zeta}\right) \operatorname{Tr}_{\mathcal{Q}_{j}}\left(\mathrm{e}^{i \phi}\right)
$$

The integral over $\zeta$ then picks out the terms with $\mathcal{A}_{1}=\mathcal{A}_{2}$, giving

$$
\left\langle\mathcal{R}_{1}\left|\delta_{M}\left(\mathrm{e}^{i \oint A}, \mathrm{e}^{i \phi}\right)\right| \mathcal{R}_{2}\right\rangle=\sum_{\mathcal{Q}_{1}, \mathcal{Q}_{2}, \mathcal{A}} \mathcal{B}_{\mathcal{A Q}_{1}}^{\mathcal{R}_{1}} \mathcal{B}_{\mathcal{A}_{2}}^{\mathcal{R}_{2}} \operatorname{Tr}_{\mathcal{Q}_{1}}\left(\mathrm{e}^{-i \phi}\right) \operatorname{Tr}_{\mathcal{Q}_{2}}\left(\mathrm{e}^{i \phi}\right)
$$

If we define the skew trace $\operatorname{Tr}_{\mathcal{R} / \mathcal{S}}$ as in (A.12), we can rewrite this as

$$
\left\langle\mathcal{R}_{1}\left|\delta_{M}\left(\mathrm{e}^{i \oint A}, \mathrm{e}^{i \phi}\right)\right| \mathcal{R}_{2}\right\rangle=\sum_{\mathcal{A}} \operatorname{Tr}_{\mathcal{R}_{1} / \mathcal{A}}\left(\mathrm{e}^{-i \phi}\right) \operatorname{Tr}_{\mathcal{R}_{2} / \mathcal{A}}\left(\mathrm{e}^{i \phi}\right)
$$

The disc. The disc of zero area gives a simple state $\Psi_{0} \in \mathcal{H}$ on its boundary,

$$
\Psi_{0}=\sum_{\mathcal{R}} S_{\mathcal{R} 0}|\mathcal{R}\rangle
$$

as was computed in ref. [6]. (This should be compared to the analogous expression in the non- $q$-deformed Yang-Mills theory - there one would have replaced $S_{\mathcal{R} 0}$ by $\operatorname{dim} \mathcal{R}$ up to overall normalization. Indeed, $S_{\mathcal{R} 0} / S_{00}$ is the quantum dimension $\operatorname{dim}_{q} \mathcal{R}$ ).

The dual Wilson line freezing operator. Also as discussed in Section 5, we need the operator $\delta_{M}\left(\mathrm{e}^{i \Phi}, \mathrm{e}^{i \phi}\right)$ which freezes $M$ of the eigenvalues of the dual Wilson line $\Phi$ at a point of $\Sigma$. The disc of zero area with this operator inserted gives a state $\Psi_{\phi} \in \mathcal{H}$ on its boundary, for which the natural formula is

$$
\Psi_{\phi}=\sum_{\mathcal{R}, \mathcal{S}} S_{\mathcal{R} \mathcal{S}} \operatorname{Tr}_{\mathcal{S} / 0}\left(\mathrm{e}^{i \phi}\right)|\mathcal{R}\rangle
$$

This is a straightforward generalization of the result of ref. [6] in the case $M=N$, along the lines of what we did above for the Wilson line freezing operator. (In the special case $M=N$, the result of ref. [6] just replaces $\operatorname{Tr}_{\mathcal{S} / 0}$ by $\operatorname{Tr}_{\mathcal{S}}$ in the above). 
The trinion (pair of pants). The trinion has three boundaries, so it gives an element in $\mathcal{H} \otimes \mathcal{H} \otimes \mathcal{H}$, namely

$$
T=\sum_{\mathcal{R}} \frac{|\mathcal{R}\rangle \otimes|\mathcal{R}\rangle \otimes|\mathcal{R}\rangle}{S_{0 \mathcal{R}}}
$$

It was computed in ref. [6]; we include it here just for completeness since it would be relevant for Riemann surfaces of genus $g>1$.

In addition to these local ingredients, we will include an overall normalization factor $Z_{\mathrm{YM}}^{0}$ in the partition function of this $q$-deformed theory; we do not give a general rule for this normalization here, but in the example we consider in the text, it can be found in (6.23).

A $q$-deformation of two-dimensional Yang-Mills theory has also been considered in ref. [31] where it was formulated using a lattice regularization. That formulation is likely to be equivalent to the one discussed here and in ref. [6]; at least the partition function appears to be the same on an arbitrary surface.

\section{Appendix C The disc wave function}

Consider the $q$-deformed 2-d Yang-Mills theory on a disc of area $p$, with parameters fixed by

$$
\theta_{\mathrm{YM}}=\theta, \quad g_{\mathrm{YM}}^{2}=g_{s}, \quad q=\mathrm{e}^{-g_{s}} .
$$

The path integral on this disc gives a state $\Psi(\xi)$ on the boundary, for which one can give a formula using the rules of Appendix B; namely, it is a sum over irreducible representations $\mathcal{R}$ of $U(N)$,

$$
\Psi(\xi)=\sum_{\mathcal{R}} S_{\mathcal{R} 0} \mathrm{e}^{-\frac{1}{2} p g_{s} C_{2}(\mathcal{R})} \mathrm{e}^{i \theta p C_{1}(\mathcal{R})} \operatorname{Tr}_{\mathcal{R}} \mathrm{e}^{i \xi}
$$

Our purpose in this section is to express this $\Psi(\xi)$ in terms of theta functions. As reviewed in Appendix A, the irreducible representations of $\mathcal{R}$ can be labeled by their highest weights $\mathcal{R}=\left(r_{1}, \ldots, r_{N}\right) \in \mathbb{Z}^{N}$, subject to the constraint $r_{1} \geq r_{2} \geq \cdots \geq r_{N}$. We also write $\hat{\mathcal{R}}=\mathcal{R}+\rho$. Now we use the Weyl character formula (A.3), the modular $S$ matrix formula (A.5), and 
the Casimirs (A.7), (A.8); altogether (C.2) becomes

$$
\begin{aligned}
\Psi(\xi)= & \sum_{\mathcal{R}}\left(\mathrm{e}^{-g_{s}\left(\|\rho\|^{2}+N / 24\right)} \sum_{\sigma \in S_{N}}(-)^{\sigma} \mathrm{e}^{g_{s}\langle\sigma(\hat{\mathcal{R}}), \rho\rangle}\right) \mathrm{e}^{-\frac{1}{2} p g_{s}\left(\|\hat{\mathcal{R}}\|^{2}-\|\rho\|^{2}\right)+i \theta p\langle\hat{\mathcal{R}}, \mathbf{1}\rangle} \\
& \times\left(D(\xi)^{-1} \sum_{\sigma^{\prime} \in S_{N}}(-)^{\sigma^{\prime}} \mathrm{e}^{i\left\langle\sigma^{\prime}(\hat{\mathcal{R}}), \xi\right\rangle}\right) .
\end{aligned}
$$

Writing $\tilde{\sigma}=\sigma \sigma^{\prime-1}$, and using the Weyl invariance of $\langle$,$\rangle and \mathbf{1}$, we can rewrite this as

$$
\begin{aligned}
\Psi(\xi)= & \mathrm{e}^{-g_{s}\left(\|\rho\|^{2}+N / 24\right)} D(\xi)^{-1} \mathrm{e}^{\frac{1}{2} p g_{s}\|\rho\|^{2}} \\
& \times \sum_{\mathcal{R}} \sum_{\sigma, \tilde{\sigma} \in S_{N}}(-)^{\tilde{\sigma}} \mathrm{e}^{-\frac{1}{2} p g_{s}\|\hat{\mathcal{R}}\|^{2}+i\left\langle\sigma(\hat{\mathcal{R}}), \tilde{\sigma}(\xi)+\theta p \mathbf{1}-i g_{s} \rho\right\rangle} .
\end{aligned}
$$

Now we want to express this as a theta function. If $\mathcal{R}$ runs over all weight vectors in a given Weyl chamber, then it is easy to see that $\hat{\mathcal{R}}$ runs over all weight vectors in the interior of that chamber. ${ }^{16}$ Since the Weyl group acts transitively to permute the Weyl chambers, the sum over $\sigma$ and $\mathcal{R}$ can be combined into a single sum over $\gamma=\sigma(\hat{\mathcal{R}})$, where $\gamma$ runs over the weight lattice $\mathbb{Z}^{N}$, or more precisely over those vectors in $\mathbb{Z}^{N}$ which are not in the boundary of any Weyl chamber. In terms of $\gamma$ the sum becomes

$$
\begin{aligned}
\Psi(\xi)= & \mathrm{e}^{-g_{s}\left(\|\rho\|^{2}+N / 24\right)} D(\xi)^{-1} \mathrm{e}^{\frac{1}{2} p g_{s}\|\rho\|^{2}} \sum_{\gamma} \mathrm{e}^{-\frac{1}{2} p g_{s}\|\gamma\|^{2}+i\left\langle\gamma, \theta p \mathbf{1}-i g_{s} \rho\right\rangle} \\
& \times \sum_{\tilde{\sigma} \in S_{N}}(-)^{\tilde{\sigma}} \mathrm{e}^{i\langle\gamma, \tilde{\sigma}(\xi)\rangle} .
\end{aligned}
$$

But now note that the sum over $\tilde{\sigma}$ vanishes if $\gamma$ is fixed by some Weyl reflection $\tilde{\sigma}$, i.e. if it lies on the boundary of a Weyl chamber. Therefore, we can extend the sum over $\gamma$ to run over the whole weight lattice $\mathbb{Z}^{N}$. The sum can be written (now dropping the ${ }^{\sim}$ on $\sigma$ for notational simplicity)

$$
\begin{aligned}
\Psi(\xi)= & \mathrm{e}^{-g_{s}\left(\|\rho\|^{2}+N / 24\right)} D(\xi)^{-1} \mathrm{e}^{\frac{1}{2} p g_{s}\|\rho\|^{2}} \\
& \times \sum_{\sigma \in S_{N}}(-)^{\sigma} \Theta_{N}\left(\frac{1}{2 \pi}\left(\sigma(\xi)+\theta p \mathbf{1}-i g_{s} \rho\right), \frac{i p g_{s}}{2 \pi}\right) .
\end{aligned}
$$

Here we have introduced the theta function of $\mathbb{Z}^{N}$,

$$
\Theta_{N}(z, \tau)=\sum_{\gamma \in \mathbb{Z}^{N}} \mathrm{e}^{\pi i \tau\|\gamma\|^{2}} \mathrm{e}^{2 \pi i\langle\gamma, z\rangle} \quad \text { for } z \in \mathbb{R}^{N}, \operatorname{Im} \tau>0,
$$

\footnotetext{
${ }^{16}$ If $N$ is even, the weight lattice has to be shifted by $\frac{1}{2} \mathbf{1}$. This subtlety modifies some of our intermediate expressions but cancels out in the final result (C.9).
} 
which obeys the functional equation

$$
\Theta_{N}(z+\tau \lambda, \tau)=\mathrm{e}^{-\pi i \tau\|\lambda\|^{2}} \mathrm{e}^{-2 \pi i\langle\lambda, z\rangle} \Theta_{N}(z, \tau) \quad \text { for } \lambda \in \mathbb{Z}^{N} .
$$

One can simplify (C.6) in the case $p=1$; namely, in this case, one can apply (C.8) with $\lambda=-\rho$. After some straightforward algebra using (A.4) the sum over $\sigma$ cancels the denominator $D(\xi)$, leaving

$$
\Psi(\xi)=\mathrm{e}^{-g_{s} N / 24} \Theta_{N}\left(\frac{1}{2 \pi}(\xi+\theta \mathbf{1}), \frac{i g_{s}}{2 \pi}\right) .
$$

\section{Appendix D Factorization}

In this appendix, we give some mathematical results which are used in the text to establish the factorization of the two-dimensional Yang-Mills amplitude with operator insertions into chiral and anti-chiral parts.

\section{D.1 Coupled representations}

An essential technical tool in studying the factorization of 2-d Yang-Mills into chiral and anti-chiral sectors, introduced in ref. [32], is the notion of a coupled representation of $U(N)$. Here we review the notion of coupled representation.

Recall that the irreducible representations of $S U(N)$ correspond to Young diagrams with no more than $N$ rows. Such a diagram can be specified by giving the lengths of the rows, $\left(\lambda_{1}, \ldots, \lambda_{N}\right)$, with $\lambda_{1} \geq \lambda_{2} \geq \cdots \geq \lambda_{N}$, and all $\lambda_{i} \geq 0$. Denote the fundamental representation by $V$. Then the representation of $S U(N)$ corresponding to $\lambda$ is obtained as a particular subspace of $V^{\otimes|\lambda|}$, roughly by symmetrizing over the rows and antisymmetrizing over the columns. In the case of $S U(N)$ one can obtain all representations in this way, but for $U(N)$ one also needs to include copies of the antifundamental representation $\bar{V}$. This corresponds to considering "extended Young diagrams" which can include "antiboxes" as well as boxes, i.e. removing the constraint that all $\lambda_{i} \geq 0$, as shown in figure 7 .

Now we can describe the coupled representations of $U(N)$. The extended Young diagram for a coupled representation $R$ is built from two Young diagrams $R_{+}, R_{-}$, just by putting boxes in the shape $R_{+}$at the upper left, antiboxes in the shape of an upside-down version of $R_{-}$at the lower right, and zero-length rows in between so that the total height of the diagram is $N$. An example is shown in figure 8 . (Note that this procedure only makes sense for sufficiently large $N$, namely, $N$ has to be greater than the combined 


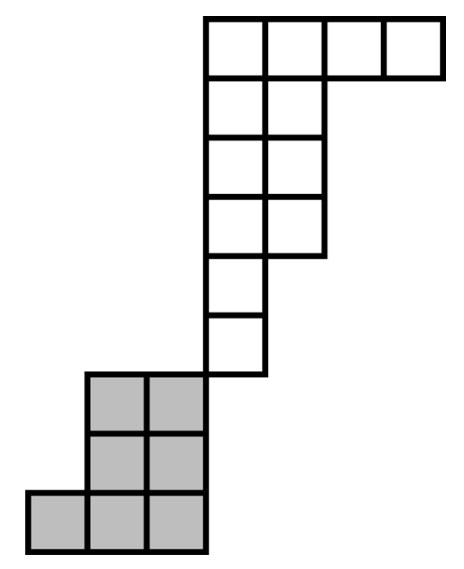

Figure 7: An extended Young diagram representing a representation of $U(N)$ (for $N=9$ ) constructed by symmetrization and antisymmetrization over both fundamental representations (white boxes) and antifundamental representations (grey boxes).

number of rows in $R_{+}$and $R_{-}$. We consider coupled representations for which one of $R_{ \pm}$has more than $\frac{1}{2} N$ rows to be undefined). We write the coupled representation $\mathcal{R}=R_{+} \overline{R_{-}}$. We will also need a slight generalization of this construction: denote by $R_{+} \overline{R_{-}}[l]$ the representation obtained by tensoring $R_{+} \overline{R_{-}}$with $l$ powers of the determinant representation of $U(N)$. This is equivalent to shifting the lengths of all rows by $l$.

The representations $R_{+} \overline{R_{-}}[l]$, where $R_{+}$and $R_{-}$are Young diagrams with $\leq \frac{1}{2} N$ rows, are a basis for the representation ring of $U(N)$ (at least for $N$ even). Another such basis would be obtained by taking instead $R_{+} \otimes \overline{R_{-}}$. The two are not the same, although $R_{+} \overline{R_{-}}$is the principal component of

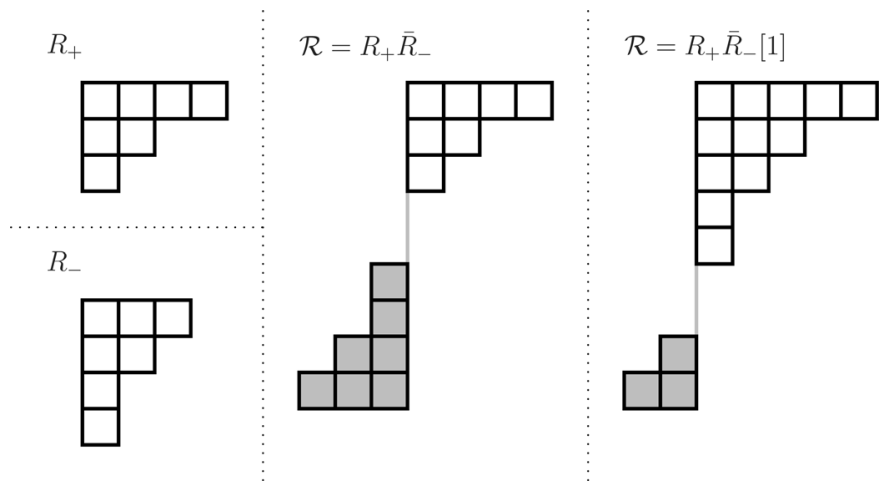

Figure 8: A coupled representation of $U(N)$ (for $N=9$ ). 
$R_{+} \otimes \overline{R_{-}} ;$the relation between the two is given by

$$
R_{+} \otimes \overline{R_{-}}=\bigoplus_{S_{ \pm}}\left[\sum_{S^{\prime}} N_{S_{+} S^{\prime}}^{R_{+}} N_{S_{-} S^{\prime}}^{R_{-}}\right] S_{+} \overline{S_{-}}
$$

(so long as $R_{ \pm}$each have $\leq \frac{1}{2} N$ rows; otherwise the right side would include $S_{+} \overline{S_{-}}$where one of $S_{ \pm}$has more than $\frac{1}{2} N$ rows, which we have not defined). Here $S^{\prime}$ and $S_{ \pm}$run over all (ordinary) Young diagrams. Note that the only $S_{+}$that contribute are ones which are contained in $R_{+}$, and similarly for $S_{-}$, so the sum in (D.1) is finite. It gives the decomposition of $R_{+} \otimes \overline{R_{-}}$ into irreducibles.

We will also need the inverse of (D.1). To get it, we use the fact that the sum over $S^{\prime}$ can be undone by summing over another auxiliary Young diagram $R^{\prime}$, using formula (A.13), here in the form

$$
\sum_{R^{\prime}, S^{\prime}}(-)^{\left|R^{\prime}\right|} N_{B_{+} R^{\prime} S^{\prime}}^{A_{+}} N_{B_{-} R^{\prime t} S^{\prime}}^{A_{-}}=\delta_{B_{+}}^{A_{+}} \delta_{B_{-}}^{A_{-}} .
$$

Applying this to (D.1) one obtains

$$
S_{+} \overline{S_{-}}=\bigoplus_{R_{ \pm}}\left(\sum_{R^{\prime}}(-)^{\left|R^{\prime}\right|} N_{R_{+} R^{\prime}}^{S_{+}} N_{R_{-} R^{\prime}}^{S_{-}}\right) R_{+} \otimes \overline{R_{-}} .
$$

Again here, $R^{\prime}$ and $R_{ \pm}$run over all ordinary Young diagrams, but only those $R_{ \pm}$which are contained in $S_{ \pm}$contribute, so the sum is finite.

One can also rewrite (D.1) in terms of characters as

$$
\begin{aligned}
& \sum_{R_{ \pm}} \operatorname{Tr}_{R_{+} \otimes \overline{R_{-}}}(U) s_{R_{+}}\left(V_{+}\right) s_{R_{-}}\left(V_{-}\right) \\
& \quad=\sum_{S_{ \pm}}\left(\sum_{S^{\prime}} s_{S_{+} \otimes S^{\prime}}\left(V_{+}\right) s_{S_{-} \otimes S^{\prime}}\left(V_{-}\right)\right) \operatorname{Tr}_{S_{+} \overline{S_{-}}}(U)
\end{aligned}
$$

and (D.3) as

$$
\begin{aligned}
& \sum_{S_{ \pm}} \operatorname{Tr}_{S_{+} \overline{S_{-}}}(U) s_{S_{+}}\left(V_{+}\right) s_{S_{-}}\left(V_{-}\right) \\
& \quad=\sum_{R_{ \pm}}\left(\sum_{R^{\prime}}(-)^{\left|R^{\prime}\right|} s_{R_{+} \otimes R^{\prime}}\left(V_{+}\right) s_{R_{-} \otimes R^{\prime \prime}}\left(V_{-}\right)\right) \operatorname{Tr}_{R_{+} \otimes \overline{R_{-}}}(U)
\end{aligned}
$$


It is useful to know how to decompose the Casimir operators for $\mathcal{R}=$ $R_{+} \overline{R_{-}}[l]$ :

$$
\begin{aligned}
& C_{1}\left(R_{+} \overline{R_{-}}[l]\right)=\left|R_{+}\right|-\left|R_{-}\right|+N l, \\
& C_{2}\left(R_{+} \overline{R_{-}}[l]\right)=\kappa_{R_{+}}+\kappa_{R_{-}}+N\left(\left|R_{+}\right|+\left|R_{-}\right|\right)+2 l\left(\left|R_{+}\right|-\left|R_{-}\right|\right)+N l^{2} .
\end{aligned}
$$

Here we introduced

$$
\kappa_{R}=\sum r_{i}^{2}-\sum c_{i}^{2}
$$

where $r_{i}$ are the lengths of the rows of the Young diagram $R$ and $c_{i}$ are the lengths of the columns. (So $\kappa_{R}=-\kappa_{R^{t}}$, where $R^{t}$ denotes the transpose of the diagram).

\section{D.2 Branching rules}

To understand the behavior of Yang-Mills theory when some eigenvalues are frozen, we need to understand the branching rules for coupled representations: how does a coupled representation of $U(N)$ decompose under $U(N) \rightarrow U\left(N_{1}\right) \times U\left(N_{2}\right)$, for $N_{1}+N_{2}=N$ ? In this section we will give formulas for these branching rules.

We begin with the case of a representation $\mathcal{R}$ of $U(N)$ which is given by an ordinary Young diagram, $\mathcal{R}=R$ (i.e. it can be constructed using only fundamental indices, without the need for antifundamentals). In this case the branching rule is well known (it is given e.g. in ref. [29] in the language of Schur functions),

$$
R \rightarrow \bigoplus_{R_{1}, R_{2}} N_{R_{1} R_{2}}^{R}\left[R_{1}, R_{2}\right]
$$

Here $R_{1}$ and $R_{2}$ run over all Young diagrams (but of course the coefficient $N_{R_{1} R_{2}}^{R}$ is only non-zero if $\left.\left|R_{1}\right|+\left|R_{2}\right|=|R|\right)$. The same rule holds for representations $\bar{R}$ constructed only from antifundamentals. Combining these two rules we can find the branching rule for tensor products,

$$
R_{+} \otimes \overline{R_{-}} \rightarrow \bigoplus_{R_{1 \pm}, R_{2 \pm}} N_{R_{1+} R_{2+}}^{R_{+}} N_{R_{1-} R_{2-}}^{R_{-}}\left[R_{1+} \otimes \overline{R_{1-}}, R_{2+} \otimes \overline{R_{2-}}\right]
$$

Now we can convert (D.10) into a branching rule for coupled representations. We start with a coupled representation $R_{+} \overline{R_{-}}$, apply (D.3) to write it in terms of tensor products, then apply (D.10) to decompose it under $U\left(N_{1}\right) \times U\left(N_{2}\right)$, then apply (D.1) to write the $U\left(N_{2}\right)$ part in terms of 
coupled representations again. This leads straightforwardly to

$$
R_{+} \overline{R_{-}} \rightarrow \bigoplus_{A_{ \pm}, Q_{ \pm}}\left(\sum_{S^{\prime}, A^{\prime}}(-)^{\left|S^{\prime}\right|} N_{A_{+} Q_{+} S^{\prime} A^{\prime}}^{R_{+}} N_{A_{-} Q_{-} S^{\prime t} A^{\prime}}^{R_{-}}\right)\left[Q_{+} \otimes \overline{Q_{-}}, A_{+} \overline{A_{-}}\right]
$$

But using (A.13) the sums over $S^{\prime}$ and $A^{\prime}$ cancel one another, and we are left with

$$
R_{+} \overline{R_{-}} \rightarrow \bigoplus_{A_{ \pm}, Q_{ \pm}}\left(N_{A_{+} Q_{+}}^{R_{+}} N_{A_{-} Q_{-}}^{R_{-}}\right)\left[Q_{+} \otimes \overline{Q_{-}}, A_{+} \overline{A_{-}}\right] .
$$

Note that for this formula to make sense we need that the $A_{+} \overline{A_{-}}$that appear are all well defined, which requires that $R_{ \pm}$are shorter than $\frac{1}{2} N_{2}$ rows.

Tensoring with powers of the determinant representation is also straightforward - writing this representation [1], it has the simple branching rule $[1] \rightarrow[[1],[1]]$. This leads to

$$
R_{+} \overline{R_{-}}[l] \rightarrow \bigoplus_{A_{ \pm}, Q_{ \pm}}\left(N_{A_{+} Q_{+}}^{R_{+}} N_{A_{-} Q_{-}}^{R_{-}}\right)\left[Q_{+} \otimes \overline{Q_{-}} \otimes[l], A_{+} \overline{A_{-}}[l]\right] .
$$

The form of (D.13) that we ultimately use will be, when $\mathcal{R}=R_{+} \overline{R_{-}}[l]$ and $\mathcal{A}=A_{+} \overline{A_{-}}[l]$,

$$
\operatorname{Tr}_{\mathcal{R} / \mathcal{A}}\left(\mathrm{e}^{i \phi}\right)=s_{R_{+} / A_{+}}\left(\mathrm{e}^{i \phi}\right) s_{R_{-} / A_{-}}\left(\mathrm{e}^{-i \phi}\right) \operatorname{det}\left(\mathrm{e}^{i l \phi}\right) .
$$

Here $\mathrm{e}^{i \phi} \in U\left(N_{1}\right)$, and we emphasize again that (D.14) holds only when $R_{ \pm}$ are shorter than $\frac{1}{2} N_{2}$ rows.

\section{D.3 Factorization of $S_{\mathcal{P Q}}$}

In order to understand the large- $N$ factorization of the $q$-deformed YangMills with insertions, we need to study the properties of the modular matrix $S_{\mathcal{P} \mathcal{Q}}$ of the $U(N)$ Chern-Simons theory in the case where $\mathcal{P}$ and $\mathcal{Q}$ are coupled representations,

$$
\begin{aligned}
& \mathcal{P}=P_{+} \overline{P_{-}}[l], \\
& \mathcal{Q}=Q_{+} \overline{Q_{-}}[m] .
\end{aligned}
$$

The most naive expectation would be that $S_{\mathcal{P} \mathcal{Q}}$ would be factorized into a piece depending on $P_{+}, Q_{+}$and a piece depending on $P_{-}, Q_{-}$. As we will 
show below, the correct formula is a sum of such terms,

$$
\begin{aligned}
S_{\mathcal{P} \mathcal{Q}} & \\
= & M(q) \eta(q)^{N} \\
& \times q^{-\frac{1}{2}\left(\kappa_{Q_{+}}+\kappa_{Q_{-}}\right)+\left(-\frac{1}{2} N-m\right)\left|P_{+}\right|+\left(-\frac{1}{2} N+m\right)\left|P_{-}\right|+\left(-\frac{1}{2} N-l\right)\left|Q_{+}\right|+\left(-\frac{1}{2} N+l\right)\left|Q_{-}\right|-2 l m N} \\
& \times \sum_{R^{\prime}}(-)^{\left|R^{\prime}\right|} q^{N\left|R^{\prime}\right|} C_{P_{+} Q_{+}^{t} R^{\prime}} C_{P_{-} Q_{-}^{t} R^{\prime t}}
\end{aligned}
$$

where $C$ is the topological vertex of ref. [20] (in canonical framing). This formula was already obtained in ref. [6], in the special case $P_{ \pm}=0$, by direct computation using results from ref. [33].

Here, we will give a physical argument which explains the reason for the factorization in the more general case of arbitrary $P_{ \pm}$and $Q_{ \pm} \cdot{ }^{17}$ We restrict to the case $l=m=0$, i.e. $\mathcal{P}=P_{+} \overline{P_{-}}$and $\mathcal{Q}=Q_{+} \overline{Q_{-}}$; the dependence on $l$ and $m$ is easily restored using (A.5) and (D.6). The idea is to realize the left side of (D.17) as the partition function of the A model topological string on the resolved conifold $T^{*} S^{3}$, with $N$ branes wrapped on $S^{3}$ and four infinite stacks of non-compact branes. Via the geometric transition of ref. [34] this is equal to the partition function of the A model on the deformed conifold $\mathcal{O}(-1) \oplus \mathcal{O}(-1) \rightarrow \mathbb{C P}^{1}$, including the four infinite stacks of non-compact branes [25]. The latter partition function can be computed using the topological vertex techniques of ref. [20], which (as we will see) gives the right side of (D.17). ${ }^{18}$

So we begin with the geometry $T^{*} S^{3}$. As in ref. [35], we represent it as a $T^{2} \times \mathbb{R}$ fibration over $\mathbb{R}^{3}$. There are two lines $l, l^{\prime}$ in $\mathbb{R}^{3}$ over which an $S^{1}$ of the $T^{2} \times \mathbb{R}$ fiber degenerates, which are shown in figure 9 . Also shown in that figure is the Lagrangian submanifold $S^{3}$, which is a $T^{2}$ fibration over a line interval connecting $l$ and $l^{\prime}$. Finally, we also indicate four Lagrangian submanifolds of $T^{*} S^{3}$, constructed as described in ref. [28]. Each such submanifold has topology $S^{1} \times \mathbb{R}^{2}$.

We will consider the topological A model on this geometry. On each Lagrangian submanifold we place an infinite stack of A model D-branes. There is then a $G L(\infty)$-valued complexified Wilson line on each stack, which couples to the open strings and thus enters the partition function. We write

\footnotetext{
${ }^{17}$ Our argument is not completely rigorous, but we hasten to add that the final result has been checked on a computer for a variety of representations $P_{ \pm}$and $Q_{ \pm}$.

${ }^{18}$ Although the geometry $\mathcal{O}(-1) \oplus \mathcal{O}(-1) \rightarrow \mathbb{C P}^{1}$ is also considered in the main text, the role the topological string is playing here is quite different from the way it appears there. We are using it here only as an auxiliary tool to prove the factorization of $S_{\mathcal{P} \mathcal{Q}}$.
} 


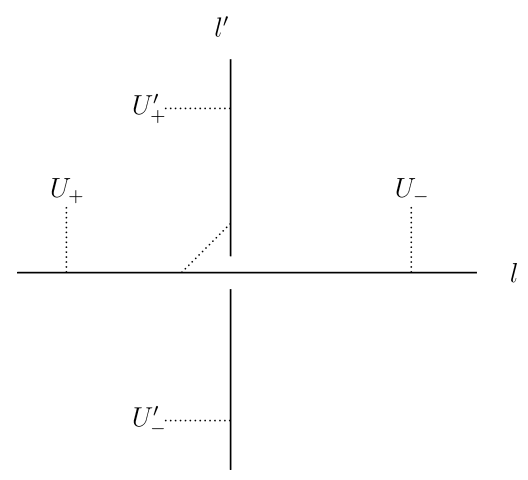

Figure 9: A toric representation of the resolved conifold geometry $T^{*} S^{3}$, with the Lagrangian submanifold $S^{3}$ indicated, as well as four non-compact Lagrangian submanifolds with topology $S^{1} \times \mathbb{R}^{2}$. Each non-compact submanifold supports an infinite stack of branes with the $G L(\infty)$-valued complexified holonomy indicated.

these four Wilson lines $U_{ \pm}$and $U_{ \pm}^{\prime}$, as indicated in the figure. We also include $N$ D-branes on the Lagrangian submanifold $S^{3}$.

The A model partition function in this geometry can be computed following refs. $[25,35]$. The closed string partition function is essentially trivial - it just gives an overall function of $q$, which is potentially ambiguous due to the non-compactness of the Calabi-Yau. We set it to 1 here. The open string partition function receives contributions from annulus diagrams running along the lines $l$ and $l^{\prime}$. On each line there are three kinds of annuli which contribute: one kind with the two boundaries on the two infinite stacks of branes, and two kinds with one boundary on an infinite stack and one boundary on the $N$ branes on $S^{3}$.

Integrating out the open string sector connecting the two infinite stacks, one gets a contribution to the partition function

$$
\sum_{R}(-)^{|R|} s_{R}\left(U_{+}\right) s_{R^{t}}\left(U_{-}\right)
$$

whereas the sectors connecting the infinite stacks to the $N$ branes on $S^{3}$ contribute operators

$$
\left(\sum_{P_{+}} s_{P_{+}}\left(U_{+}\right) \operatorname{Tr}_{P_{+}}(V)\right)\left(\sum_{P_{-}} s_{P_{-}}\left(U_{-}\right) \operatorname{Tr}_{P_{-}}\left(V^{\dagger}\right)\right)
$$

with $V$ representing the holonomy around the $S^{1}$ where the annuli over $l$ meet $S^{3}$. 
Combining (D.18) and (D.19), one obtains for the open string contribution from the branes on $l$

$$
\sum_{R, P_{ \pm}}(-)^{|R|} s_{R \otimes P_{+}}\left(U_{+}\right) s_{R^{t} \otimes P_{-}}\left(U_{-}\right) \operatorname{Tr}_{\mathcal{P}}(V) .
$$

Using the formula (D.5), (D.20) becomes

$$
\sum_{P_{ \pm}} s_{P_{+}}\left(U_{+}\right) s_{P_{-}}\left(U_{-}\right) \operatorname{Tr}_{\mathcal{P}}(V) .
$$

Similarly, from the branes on $l^{\prime}$ we obtain

$$
\sum_{Q_{ \pm}} s_{Q_{+}}\left(U_{+}^{\prime}\right) s_{Q_{-}}\left(U_{-}^{\prime}\right) \operatorname{Tr}_{\mathcal{Q}}\left(V^{\prime}\right)
$$

where $V^{\prime}$ is the holonomy on the $S^{1}$ where the annuli over $l^{\prime}$ meet $S^{3}$. Altogether, then, the contribution from open strings which involve the four infinite stacks of branes is

$$
\sum_{P_{ \pm}, Q_{ \pm}}\left[s_{P_{+}}\left(U_{+}\right) s_{P_{-}}\left(U_{-}\right) s_{Q_{+}}\left(U_{+}^{\prime}\right) s_{Q_{-}}\left(U_{-}^{\prime}\right)\right] \operatorname{Tr}_{\mathcal{P}}(V) \operatorname{Tr}_{\mathcal{Q}}\left(V^{\prime}\right) .
$$

We view this $s_{P}(V) s_{Q}\left(V^{\prime}\right)$ as a product of Wilson line operators deforming the open string theory on the $N$ branes on $S^{3}$, namely the $U(N)$ ChernSimons theory; these operators are arranged so as to give a Hopf link in $S^{3}$. The Chern-Simons amplitude with this link inserted is simply $S_{\mathcal{P} \mathcal{Q}}[36]$, so the topological string partition function is finally

$$
\psi_{\text {top }}=\sum_{P_{ \pm}, Q_{ \pm}}\left[s_{P_{+}}\left(U_{+}\right) s_{P_{-}}\left(U_{-}\right) s_{Q_{+}}\left(U_{+}^{\prime}\right) s_{Q_{-}}\left(U_{-}^{\prime}\right)\right] S_{\mathcal{P} \mathcal{Q}}
$$

To get the desired factorization of $S_{\mathcal{P} \mathcal{Q}}$ we now compute this partition function in another way: namely, we consider the geometric transition [34] to the deformed conifold $\mathcal{O}(-1) \oplus \mathcal{O}(-1) \rightarrow \mathbb{C P}^{1}$, with the volume of $\mathbb{C P}^{1}$ given by $t=N g_{s}$, and with four infinite stacks of branes, as shown in figure 10 . Using the topological vertex of ref. [20], the A model partition function in this geometry can be computed as ${ }^{19}$

$$
\begin{aligned}
\psi_{\text {top }}= & M(q) \eta(q)^{N} \sum_{P_{ \pm}, Q_{ \pm}}\left[s_{P_{+}}\left(U_{+}\right) s_{P_{-}}\left(U_{-}\right) s_{Q_{+}}\left(U_{+}^{\prime}\right) s_{Q_{-}}\left(U_{-}^{\prime}\right)\right] \\
& \times q^{-\frac{1}{2}\left(\kappa(Q+)+\kappa\left(Q_{-}\right)+N\left(\left|P_{+}\right|+\left|Q_{+}\right|+\left|P_{-}\right|+\left|Q_{-}\right|\right)\right)} \\
& \times \sum_{R^{\prime}}(-)^{\left|R^{\prime}\right|} q^{N\left|R^{\prime}\right|} C_{P_{+} Q_{+}^{t} R^{\prime}} C_{P_{-} Q_{-}^{t} R^{\prime t}}
\end{aligned}
$$

More precisely, the factors $M(q) \eta(q)^{N}$ in ref. (D.25) do not appear in ref. [20], so they deserve some extra comment. The factor $M(q)$ is associated with

\footnotetext{
${ }^{19}$ One could determine the framing factors in (D.25) from first principles; we determined them by requiring that the large $N$ limit of our factorization formula be correct.
} 


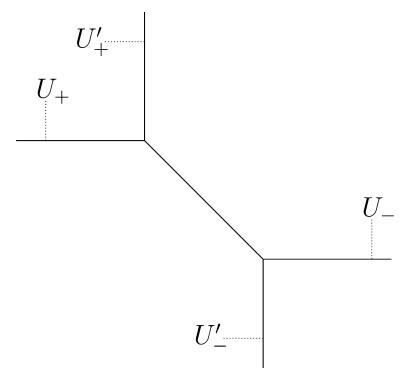

Figure 10: The geometry of figure 9 after the geometric transition.

the closed string sector; namely, in the large volume limit, it was shown in refs. $[37,38]$ that the closed A model partition function reduces to $M(q)^{\chi / 2}$ on a Calabi-Yau three-fold with Euler characteristic $\chi$. In the non-compact case we are considering here $\chi$ is ambiguous, but the change in $\chi$ that occurs due to the geometric transition would naturally be $\Delta \chi=2$ (a 3-cycle gets replaced by a 2-cycle). Thus, since we took $\chi=0$ before the transition (we chose the closed string contribution in $\psi_{\text {top }}$ to be 1 ), we should take $\chi=2$ after the transition, which accounts for the factor $M(q)$. The factor $\eta(q)^{N}$ is not as easily understood, but is presumably associated with the fact that $N$ D3-branes have disappeared in the transition; the same factor appeared in ref. [39] associated to a single non-compact D3-brane. Comparing (D.24) and (D.25) one obtains the desired formula (D.17).

One can also compute a factorization formula for $1 / S_{0 \mathcal{P}}$, as was done in ref. [6]:

$$
\frac{1}{S_{0 \mathcal{P}}}=M(q)^{-1} \eta(q)^{-N} q^{\frac{1}{2} N\left(\left|P_{+}\right|+\left|P_{-}\right|\right)} \frac{\sum_{R} C_{P_{+} 0 R} q^{N|R|} C_{P_{-} 0 R}}{C_{00 P_{+}}^{2} C_{00 P_{-}}^{2}} .
$$

It would be interesting to know whether there is a physical argument for this factorization formula along the lines of the argument given above for (D.17).

\section{D.4 Factorization of $q$-deformed Yang-Mills amplitudes}

Now we are ready to consider the large $N$ factorization of the $q$-deformed Yang-Mills amplitudes with operator insertions. We will approach the problem by factorizing each of the elementary ingredients from Appendix B separately.

In the large $N$ ('t Hooft) limit, a convenient basis for the Hilbert space $\mathcal{H}$ is given by the characters of the "coupled representations" which we introduced 
in Appendix $\mathrm{A}$; we write $\mathcal{R}=\left|R_{+} \overline{R_{-}}[l]\right\rangle$ for the states corresponding to the coupled representations. As was argued in ref. [32], these representations are the only ones which contribute to the large $N$ amplitudes, to all orders in $1 / N$; the reason is that they are the only ones with $C_{2}(\mathcal{R}) \sim N$. All other representations are exponentially suppressed in the 't Hooft limit by the factors $\mathrm{e}^{-\frac{1}{2} a g_{\mathrm{YM}}^{2} C_{2}(\mathcal{R})}$, which appear on a surface of area $a$ as in Appendix Bin the large $N$ limit they give contributions which are $\mathcal{O}\left(\mathrm{e}^{-N}\right)$.

In this factorized basis, the ingredients of the amplitudes can be written as follows:

The annulus. Using (B.7) together with (D.6) and (D.7), we obtain easily

$$
\begin{aligned}
\left\langle R_{1+}\right. & \left.\overline{R_{1-}}\left[l_{1}\right]\left|A_{a}\right| R_{2+} \overline{R_{2-}}\left[l_{2}\right]\right\rangle \\
= & \delta_{R_{1+}} R_{2+} \delta_{R_{1-}} R_{2-} \delta_{l_{1} l_{2}} \\
& \times \mathrm{e}^{-a \frac{1}{2} g_{\mathrm{YM}}^{2} N l^{2}} \mathrm{e}^{i N a \theta_{\mathrm{YM}} l} \mathrm{e}^{-a\left(\frac{1}{2} g_{\mathrm{YM}}^{2}\left(\kappa_{R_{+}}+N\left|R_{+}\right|+2 l\left|R_{+}\right|\right)-i \theta_{\mathrm{YM}}\left|R_{+}\right|\right)} \\
& \times \mathrm{e}^{-a\left(\frac{1}{2} g_{\mathrm{YM}}^{2}\left(\kappa_{R_{-}}+N\left|R_{-}\right|-2 l\left|R_{-}\right|\right)+i \theta_{\mathrm{YM}}\left|R_{-}\right|\right)}
\end{aligned}
$$

The Wilson line freezing operator. From (B.11) and (D.14) we find

$$
\begin{aligned}
\left\langle R_{1+} \overline{R_{1-}}\left[l_{1}\right]\left|\delta_{M}\left(\mathrm{e}^{i \oint A}, \mathrm{e}^{i \phi}\right)\right| R_{2+} \overline{R_{2-}}\left[l_{2}\right]\right\rangle \\
=\delta_{l_{1}, l_{2}}\left(\sum_{A_{+}} s_{R_{1+} / A_{+}}\left(\mathrm{e}^{-i \phi}\right) s_{R_{2+} / A_{+}}\left(\mathrm{e}^{i \phi}\right)\right) \\
\quad \times\left(\sum_{A_{-}} s_{R_{1-} / A_{-}}\left(\mathrm{e}^{i \phi}\right) s_{R_{2-} / A_{-}}\left(\mathrm{e}^{-i \phi}\right)\right) .
\end{aligned}
$$

The disc. From (B.12) and (D.17) this is

$$
\begin{aligned}
\Psi_{0}= & M(q) \eta(q)^{N} \sum_{l \in \mathbb{Z}, R_{ \pm}} q^{-\frac{1}{2} N\left(\left|R_{+}\right|+\left|R_{-}\right|\right)}\left[\sum_{R^{\prime}}(-)^{\left|R^{\prime}\right|} q^{N\left|R^{\prime}\right|} C_{R_{+} 0 R^{\prime}} C_{R_{-} 0 R^{\prime t}}\right] \\
& \times\left|R_{+} \overline{R_{-}}[l]\right\rangle .
\end{aligned}
$$


The dual Wilson line freezing operator. From (B.13), (D.17) and (D.14) we get

$$
\begin{aligned}
\Psi_{\phi} & \\
= & M(q) \eta(q)^{N} \sum_{l, m \in \mathbb{Z}, R_{ \pm}, S_{ \pm}} \\
& \times q^{-\frac{1}{2}\left(\kappa_{S_{+}}+\kappa_{S_{-}}\right)+\left(-\frac{1}{2} N-m\right)\left|R_{+}\right|+\left(-\frac{1}{2} N+m\right)\left|R_{-}\right|+\left(-\frac{1}{2} N-l\right)\left|S_{+}\right|+\left(-\frac{1}{2} N+l\right)\left|S_{-}\right|-2 l m N} \\
& \times\left[\sum_{R^{\prime}}(-)^{\left|R^{\prime}\right|} q^{N\left|R^{\prime}\right|} C_{R_{+} S_{+}^{t} R^{\prime}} C_{R_{-} S_{-}^{t}} R^{\prime t}\right] s_{S_{+}}\left(\mathrm{e}^{-i \phi}\right) s_{S_{-}}\left(\mathrm{e}^{i \phi}\right) \operatorname{det}\left(\mathrm{e}^{i m \phi}\right)\left|R_{+} \overline{R_{-}}[l]\right\rangle .
\end{aligned}
$$

The trinion (pair of pants). From (B.14) and (D.26) this is

$$
\begin{aligned}
T= & \sum_{l \in \mathbb{Z}, R_{ \pm}}\left[\sum_{R^{\prime}} q^{\frac{1}{2} N\left(\left|R_{+}\right|+\left|R_{-}\right|\right)} \frac{C_{R_{+} 0 R^{\prime}} q^{N\left|R^{\prime}\right|} C_{R_{-} 0 R^{\prime}}}{C_{00 R_{+}}^{2} C_{00 R_{-}}^{2}}\right] \\
& \times\left|R_{+} \overline{R_{-}}[l]\right\rangle \otimes\left|R_{+} \overline{R_{-}}[l]\right\rangle \otimes\left|R_{+} \overline{R_{-}}[l]\right\rangle .
\end{aligned}
$$

\section{References}

[1] B. de Wit, G. Lopes Cardoso and T. Mohaupt, Corrections to macroscopic supersymmetric black-hole entropy, Phys. Lett. B451, (1999), 309-316, hep-th/9812082.

[2] B. de Wit, G. Lopes Cardoso and T. Mohaupt, Deviations from the area law for supersymmetric black holes, Fortsch. Phys. 48 (2000), 49-64, hep-th/9904005.

[3] B. de Wit, G. Lopes Cardoso and T. Mohaupt, Macroscopic entropy formulae and non-holomorphic corrections for supersymmetric black holes, Nucl. Phys. B567 (2000), 87-110, hep-th/9906094.

[4] H. Ooguri, A. Strominger and C. Vafa, Black hole attractors and the topological string, Phys. Rev. D70 (2004), 106007, hep-th/0405146.

[5] C. Vafa, Two dimensional Yang-Mills, black holes and topological strings, hep-th/0406058.

[6] M. Aganagic, H. Ooguri, N. Saulina and C. Vafa, Black holes, qdeformed 2d Yang-Mills, and non-perturbative topological strings, Nucl. Phys. B715 (2005), 304-348, hep-th/0411280.

[7] A. Dabholkar, Exact counting of black hole microstates, Phys. Rev. Lett. 94 (2005), 241-301, hep-th/0409148.

[8] A. Sen, Black holes, elementary strings and holomorphic anomaly, JHEP 0507 (2005), 063, hep-th/0502126. 
[9] A. Dabholkar, F. Denef, G.W. Moore and B. Pioline, Exact and asymptotic degeneracies of small black holes, JHEP 0508 (2005), 021, hep-th/0502157.

[10] A. Sen, Black holes and the spectrum of half-BPS states in $\mathcal{N}=4$ supersymmetric string theory, Adv. Theor. Math. Phys. 9 (2005), 527-558, hep-th/0504005.

[11] S. Ferrara, R. Kallosh and A. Strominger, $\mathcal{N}=2$ extremal black holes, Phys. Rev. D52 (1995), 5412-5416, hep-th/9508072.

[12] A. Strominger, Macroscopic entropy of $\mathcal{N}=2$ extremal black holes, Phys. Lett. B383 (1996), 39-43, hep-th/9602111.

[13] E. Witten, Quantum background independence in string theory, hep-th/9306122.

[14] R. Dijkgraaf, E. Verlinde and M. Vonk, On the partition sum of the NSs five-brane, hep-th/0205281.

[15] M. Bershadsky, S. Cecotti, H. Ooguri and C. Vafa, Holomorphic anomalies in topological field theories, Nucl. Phys. B405 (1993), 279-304, hep-th/9302103.

[16] M. Bershadsky, S. Cecotti, H. Ooguri and C. Vafa, Kodaira-Spencer theory of gravity and exact results for quantum string amplitudes, Commun. Math. Phys. 165 (1994), 311-428, hep-th/9309140.

[17] E. Verlinde, Attractors and the holomorphic anomaly, hep-th/0412139.

[18] M. Bershadsky, V. Sadov and C. Vafa, D-branes and topological field theories, Nucl. Phys. B463 (1996), 420-434, hep-th/9511222.

[19] J. Bryan and R. Pandharipande, The local Gromov-Witten theory of curves, math.ag/0411037.

[20] M. Aganagic, A. Klemm, M. Marino and C. Vafa, The topological vertex, Commun. Math. Phys. 254 (2005), 425-478, hep-th/0305132.

[21] M. Aganagic, R. Dijkgraaf, A. Klemm, M. Marino and C. Vafa, Topological strings and integrable hierarchies, Commun. Math. Phys. 261 (2006), 451-516, hep-th/0312085.

[22] S. Elitzur, G.W. Moore, A. Schwimmer and N. Seiberg, Remarks on the canonical quantization of the Chern-Simons-Witten theory, Nucl. Phys. B326 (1989), 108.

[23] M. Aganagic, A. Klemm, M. Marino and C. Vafa, Matrix model as a mirror of Chern-Simons theory, JHEP 0402 (2004), 010, hep-th/0211098.

[24] E. Witten, Chern-Simons gauge theory as a string theory, Prog. Math. 133 (1995), 637-678, hep-th/9207094.

[25] H. Ooguri and C. Vafa, Knot invariants and topological strings, Nucl. Phys. B577 (2000), 419-438, hep-th/9912123. 
[26] R.C. McLean, Deformations of calibrated submanifolds, Comm. Anal. Geom. 6 no. 4, (1998), 705-747.

[27] M. Aganagic, A. Klemm and C. Vafa, Disk instantons, mirror symmetry and the duality web, Z. Naturforsch. A57 (2002), 1-28, hep-th/0105045.

[28] M. Aganagic and C. Vafa, Mirror symmetry, D-branes and counting holomorphic discs, hep-th/0012041.

[29] I.G. Macdonald, Symmetric Functions and Hall Polynomials, Oxford Mathematical Monographs, second ed., The Clarendon Press Oxford University Press, New York, 1995 (with contributions by A. Zelevinsky, Oxford Science Publications).

[30] S. Cordes, G.W. Moore and S. Ramgoolam, Lectures on 2-d YangMills theory, equivariant cohomology and topological field theories, Nucl. Phys. Proc. Suppl. 41 (1995), 184-244, hep-th/9411210.

[31] E. Buffenoir and P. Roche, Two-dimensional lattice gauge theory based on a quantum group, Commun. Math. Phys. 170 (1995), 669-698, hep-th/9405126.

[32] D.J. Gross and W. Taylor, Two-dimensional QCD is a string theory, Nucl. Phys. B400 (1993), 181-210, hep-th/9301068.

[33] A. Iqbal and A.-K. Kashani-Poor, The vertex on a strip, hep-th/0410174.

[34] R. Gopakumar and C. Vafa, On the gauge theory/geometry correspondence, Adv. Theor. Math. Phys. 3 (1999), 1415-1443, hep-th/9811131.

[35] M. Aganagic, M. Marino and C. Vafa, All loop topological string amplitudes from Chern-Simons theory, Commun. Math. Phys. 247 (2004), 467-512, hep-th/0206164.

[36] E. Witten, Quantum field theory and the Jones polynomial, Commun. Math. Phys. 121 (1989), 351.

[37] R. Gopakumar and C. Vafa, M-theory and topological strings I, hep-th/9809187.

[38] C. Faber and R. Pandharipande, Logarithmic series and Hodge integrals in the tautological ring (with an appendix by D. Zagier), Michigan Math. J. 48 (2000), 215-252, math.ag/0002112.

[39] N. Saulina and C. Vafa, D-branes as defects in the Calabi-Yau crystal, hep-th/0404246. 